\title{
3 Von den geschlossenen Fürsorgeheimen für Geschlechtskranke in der SBZ zu den geschlossenen Venerologischen Stationen in der DDR
}

\subsection{Die SMAD-Befehle zur „Bekämpfung von Geschlechtskrankheiten“ in der SBZ und der DDR}

Nach dem Ende des Zweiten Weltkriegs versuchten die alliierten Truppen, eine schnelle Ausbreitung von Infektionskrankheiten in den Besatzungszonen einzudämmen. ${ }^{55}$ Entsprechend trafen sie Maßnahmen, um Ansteckungen zu verhindern. Vor allem die Bekämpfung von Geschlechtskrankheiten hatte bei den Alliierten eine hohe Priorität, da sie fürchteten, dass die deutschen Verwaltungen und Ärzte die immer bedrohlicher werdende Zahl an Infektionen nicht beherrschen würden. Die SMAD reagierte in der SBZ rasch und griff rigoros durch. So wurde zwischen 1945 und 1947 eine dichte Folge von SMAD-Befehlen erlassen, welche die Aktivierung und Durchführung von einheitlichen Vorgehensweisen bei der Bekämpfung und Verhinderung von sexuell übertragbaren Krankheiten in der SBZ und der DDR regelte. Die SMAD-Befehle zur Bekämpfung und Eindämmung von Geschlechtskrankheiten waren Übersetzungen von Regelungen aus der sowjetischen Medizinalpraxis. ${ }^{56}$ Vor allem der SMAD-Befehl Nr. 273, gültig bis 1961, bildete bis zu diesem Zeitpunkt die rechtliche Grundlage für Zwangseinweisungen in die geschlossenen Venerologischen Stationen.

55 Brinkschulte: Bordellbaracken für die Besatzungstruppen (Anm. 36), S. 253.

56 Krumbiegel: Polikliniken in der SBZ, DDR (Anm. 45), S. 168. 
In der SBZ wurde das nach wie vor gültige „Gesetz zur Bekämpfung der Geschlechtskrankheiten" vom 18. Februar 1927 als unzureichend betrachtet, um einen wirkungsvollen Beitrag zur Bekämpfung und Eindämmung von Geschlechtskrankheiten zu leisten. ${ }^{57}$ Daher wurde bereits in den ersten Monaten der sowjetischen Besatzungszeit mit dem SMAD-Befehl Nr. 25 ,Über die Maßnahmen zur Bekämpfung der Geschlechtskrankheiten in der sowjetischen Besatzungszone Deutschlands "58 vom 7. August 1945 eine Anordnung zur Vorbeugung, medizinischen Versorgung sowie zur Zwangsbehandlung getroffen. Mit dem Befehl wurden unter anderem die Einrichtung eines dichten Netzes von medizinischen Anstalten sowie die Ausbildung von Venerologen geregelt. ${ }^{59}$ Darüber hinaus bot der SMAD-Befehl Nr. 25 Mittel, um Zwangshospitalisierungen durchzusetzen und einen energischen Kampf gegen die Prostitution zu führen. ${ }^{60} \mathrm{Im} \$ 3$ hieß es, dass „,so viele Betten in Spezialkliniken und Krankenabteilungen bereitzustellen“ seien, dass „alle Fälle ansteckendender Formen von Geschlechtskrankheiten einer Hospitalisierung “ zugeführt werden können, „wobei alle ansteckenden Stadien der Syphilis zwangsweiser Hospitalisierung“ unterlagen. Der $\mathbb{5} 5$ sah die „Unterbindung der legalen und illegalen Prostitution“ vor. „Geschlechtskranke Prostituierte [erfuhren] Zwangsbehandlung. "Auf diese Weise ermöglichte der Befehl vor allem die Sanktionierung der Prostitution in der SBZ. Schließlich war im $\mathbb{} 7$ die „obligatorische Registrierung von Geschlechtskranken in amtlichen Behandlungsstellen und Privatpraxen" vorgesehen. ${ }^{61}$

Aufgrund der mangelhaften Durchführung des SMAD-Befehls Nr. 25 in der gesamten SBZ wurde in rascher Folge ein weiterer Befehl von der SMAD erlassen. Dieser SMAD-Befehl Nr. 30 trat am 12. Februar 1946 in Kraft und wurde im Juli 1946 durch ausführende Bestimmungen der „Deutschen Zentralverwaltung für das Gesundheitswesen“ (DZVG) ergänzt. ${ }^{62}$ Darin war die Einrichtung von Ambulatorien vorgesehen, in denen die Beratungen und Behandlungen von Geschlechtskrankheiten ebenso durchgeführt werden sollten wie in allen Krankenhäusern und Privatpraxen. Gleichzeitig wurde befohlen, ein Netz von Beratungsstellen bzw. Fürsorgestellen für Geschlechtskranke zur prophylaktischen Behandlung und Untersuchung einzurichten. Zugleich verfügte die DZVG, dass die Beratungs- und Behandlungsstellen für Geschlechtskranke (Ambulatorien) durch Mittel der DZVG bzw. der Provinzialverwaltun-

57 Elste G (1967) Die SMAD-Befehle 25, 030 und 273 - ihre Bedeutung für die Verhütung und Bekämpfung der Geschlechtskrankheiten während des Aufbaus des antifaschistisch-demokratischen Gesundheitswesens von 1945 bis zur Gründung der Deutschen Demokratischen Republik. In: Ministerrat der Deutschen Demokratischen Republik, Ministerium für Gesundheitswesen (Hrsg.) Die Bedeutung der Befehle der SMAD für den Aufbau des sozialistischen Gesundheitswesens der Deutschen Demokratischen Republik. 61-71. Ministerrat der DDR, Ministerium für Gesundheitswesen Berlin.

58 Elste: Die SMAD-Befehle 25, 030 und 273 - ihre Bedeutung (Anm. 57), S. 62. 
3.1 Die SMAD-Befehle zur „Bekämpfung von Geschlechtskrankheiten“ in der SBZ und der DDR

gen finanziert wurden. Diese finanzielle Absicherung der Ambulatorien sollte die kostenlose Inanspruchnahme vor allem der prophylaktischen Angebote durch die Bevölkerung dauerhaft sichern. ${ }^{63}$

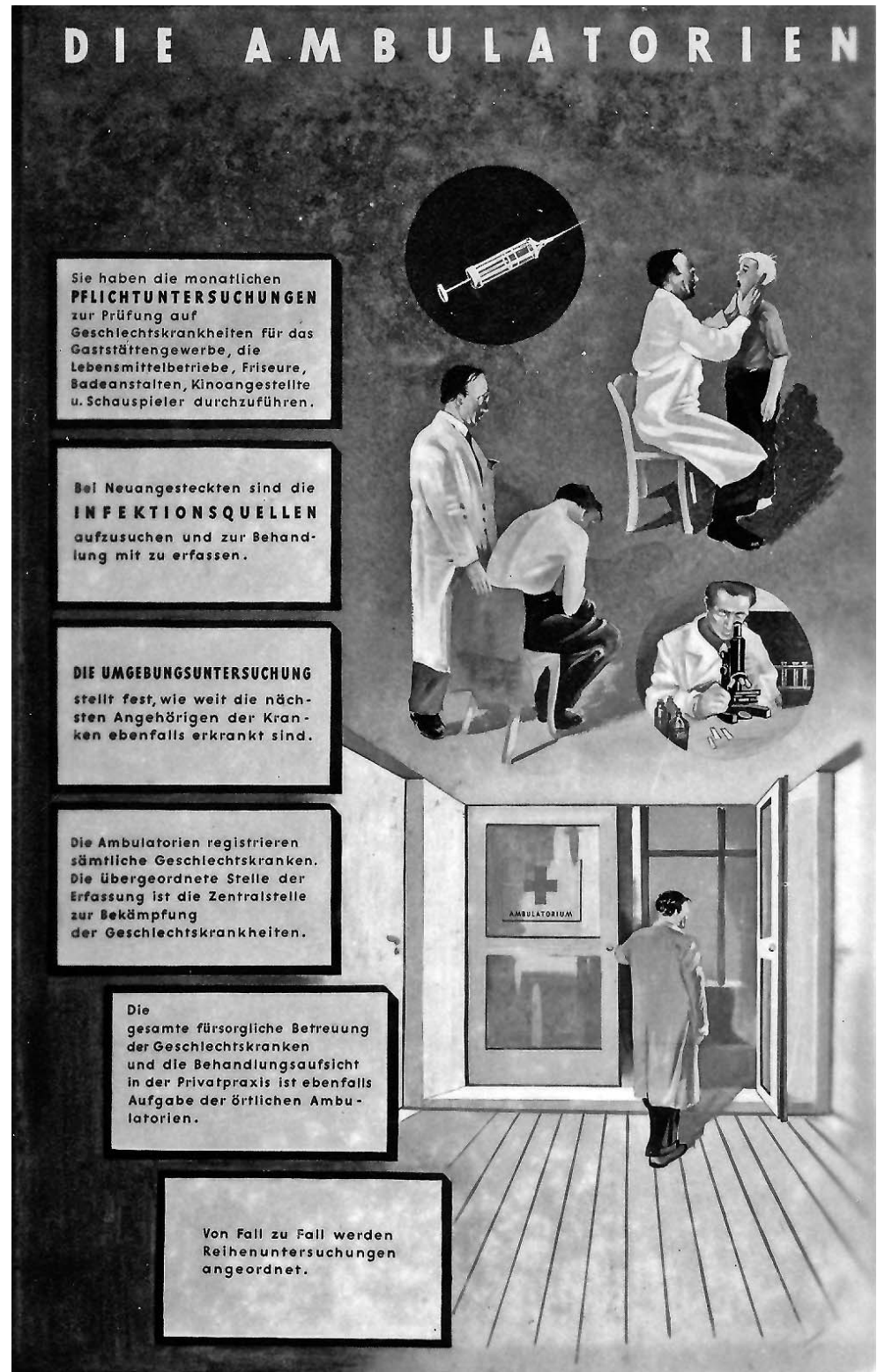

Abb. 2 „Die Ambulatorien“; Aufgaben der Ambulatorien für Geschlechtskrankheiten (Plakat um 1948)

63 Krumbiegel: Polikliniken in der SBZ, DDR (Anm. 45), S. 171. 
3 Von den geschlossenen Fürsorgeheimen für Geschlechtskranke in der SBZ zu den geschlossenen Venerologischen Stationen in der DDR

Darüber hinaus wurde im $\mathbb{} 7$ des SMAD-Befehls Nr. 30 geregelt, dass bis zum 1. März 1946 in jedem Bezirk und in der Stadt Berlin „Fürsorgeheime mit polizeilichem Schutz für die zwangsmässige Behandlung“ eingerichtet werden. Diese Fürsorgeheime dienten der obligatorischen Behandlung „derjenigen Personen, die sich der Behandlung der Syphilis und Tripper entziehen, derjenigen kranken Frauen, die sich mit Prostitution befassen und derjenigen Frauen, die Militärangehörige angesteckt haben“. Die zwangsmäßig Behandelten sollten nach ihrer Entlassung eine Geldstrafe von 200 Reichsmark zahlen oder innerhalb eines Monats zur Zwangsarbeit herangezogen werden. Die Funktion der Fürsorgeheime wurde im $₫ 8$ festgeschrieben: „In den Fürsorgeheimen sollen die eingelieferten Personen handwerklich beschäftigt werden, nach ihrer Entlassung sollen die angefertigten Objekte zurückgelassen werden und die weitere Führung der geheilten Frauen überwacht werden." Schließlich wurde im $\$ 10$ festgelegt, dass in „,den Kliniken für Haut- und Geschlechtskrankheiten der Städte Berlin, Halle, Leipzig, Dresden, Rostock und Greifswald (...) die Anlernung von Ärzten für Geschlechtskrankheiten aus der Zahl der allgemeinen Ärzte in 6-wöchigen Kursen von 20 Personen in jeder Klinik vorzunehmen" ist. ${ }^{64}$

Die SMAD-Befehle Nr. 25 und 30 standen im Wesentlichen für den flächendeckenden Aufbau von Einrichtungen zur Vorbeugung und Behandlung von Geschlechtskrankheiten, der Einführung von Landes-, Bezirks- und Kreisvenerologen, der Bildung von Sonderabteilungen an Gesundheitsämtern, den Aufbau eines Meldesystems und Berichtswesens für Geschlechtskrankheiten sowie die Durchführung von Reihenuntersuchungen unter anderem bei Beschäftigten in der Lebensmittelindustrie. Es wurden die rechtlichen Rahmenbedingungen für die Isolierung von Patienten mit infektiösen Formen von Geschlechtskrankheiten, die Pflicht des Erkrankten, sich behandeln zu lassen und die Festlegung vielfältiger Bestrafungsvorschriften geregelt. Zudem sollte die Prostitution eingedämmt, breit angelegte Aufklärungsmaßnahmen für die Bevölkerung der SBZ ausgearbeitet sowie Venerologen ausgebildet werden. In der Summe fallen vier Aspekte besonders auf, die mit den SMAD-Befehlen Nr. 25 und 30 verfolgt werden sollten: 1. Die Einführung der namentlichen Meldepflicht für Geschlechtskranke. 2. Die Ausbildung von Hilfsvenerologen. 3. Die Zwangshospitalisierung aller ansteckenden Formen der Syphilis. 4. Die Beratung, Aufklärung und gesundheitliche Erziehung der Bevölkerung.

Nachdem mit diesen SMAD-Befehlen das grundlegende Vorgehen bei der Bekämpfung von Geschlechtskrankheiten implementiert war, folgte der SMADBefehl Nr. 273 „Verordnung zur Bekämpfung der Geschlechtskrankheiten unter der deutschen Bevölkerung in der sowjetischen Besatzungszone Deutsch-

64 StAZ: R3/1032. Fürsorgeheim Schloß Osterstein: Befehl des Obersten Chefs der Sowjetischen Militäradministration des Oberkommandierenden der Gruppe der sowjetischen Besatzungsarmee, Marschall Schukow, in Deutschland 12. Februar 1946, Nr. 030, Bl. 6. 
lands“65 vom 11. Dezember 1947. Der SMAD-Befehl Nr. 273, der bis 1961 die Einweisungen in die geschlossenen Venerologischen Stationen regelte, nimmt in zweifacher Weise eine besondere Stellung bei der Bekämpfung von Geschlechtskrankheiten ein. Zum einen wurden mit ihm die deutschen Bestimmungen des „Gesetzes zur Bekämpfung von Geschlechtskrankheiten“ vom 18. Februar 1927 aufgehoben. ${ }^{66}$ Zum anderen wurden mit dem Befehl einzelne Straftatbestände und die darauf folgenden Strafen sowie Rechte und Pflichten für Ärzte und Gesundheitsämter definiert. So heißt es unter anderem im $\mathbb{2 3}$ : Gehört jemand „zu den Personen, die häufig wechselnden Geschlechtsverkehr unterhalten, so kann das Gericht neben der Strafe seine Unterbringung in einem Arbeitshaus anordnen“. ${ }^{67}$

Den Ärzten wurden unter anderem folgende Pflichten auferlegt: Aufklärungsund Belehrungspflicht gegenüber seinen Patienten (\$ 8), Anzeigepflicht von Geschlechtskrankheiten (\$ 9) oder Befragungspflicht gegenüber einer krankheitsverdächtigen Person (\$11). Sollten die Ärzte diesen Pflichten nicht nachkommen, drohten ihnen Geldstrafen bis zu 3.0oo Reichsmark bzw. Gefängnis bis zu einem Jahr, wie es im $\mathbb{s} 16$ heißt. ${ }^{68}$ Die Gesundheitsämter wiederum erhielten weitreichende Rechte aber auch Pflichten: Erteilung von Berufsverboten für Geschlechtskranke ( $\$ 17)$, Anordnung periodischer Untersuchungen für Geschlechtskranke oder Personen mit häufig wechselnden Geschlechtspartnern ( $\$ 18)$ oder die sofortige Unterbringung in einem geschlossenen Krankenhaus ( $\$ 14),{ }^{69}$,wenn a) er die Anordnungen des Arztes nicht befolgt oder sich der Behandlung entzieht, b) er entgegen dem Verbot des $\mathbb{2}$ Absatz 1C [Enthaltung von Geschlechtsverkehr] geschlechtlich verkehrt, c) auf Grund seiner Lebensweise anzunehmen ist, daß er die Geschlechtskrankheit weiterverbreitet, d) er trotz entsprechender Anordnung des Arztes kein Krankenhaus aufsucht oder das Krankenhaus vorzeitig verläßt“.70

Mit den geschlossenen Krankenhäusern waren die geschlossenen Venerologischen Stationen, Abteilungen und Krankenstationen gemeint. Diese Einrichtungen wurden, parallel zu den Fürsorgeheim für Geschlechtskranke, seit in Kraft treten der SMAD-Befehle Nr. 30 und 273 in der SBZ eingerichtet. Mit den SMAD-Befehlen wurde die rechtliche Grundlage für den Umgang mit Geschlechtskranken gelegt, die bis in die 196oer Jahre der DDR hineinreichte. Die Befehle begründeten in der SBZ eine langanhaltende Tradition im Umgang

65 Befehl Nr. 273 des Obersten Chefs der Sowjetischen Militär-Administration in Deutschland über die Bekämpfung der Geschlechtskrankheiten unter der deutschen Bevölkerung in der sowjetischen Besatzungszone Deutschlands vom 11. Dezember 1947. In: Deutsche Zentralverwaltung für das Gesundheitswesen (Hrsg.) Die gesetzlichen Bestimmungen zur Bekämpfung der Geschlechtskrankheiten in der sowjetischen Besatzungszone Deutschlands. 13-22. Taschenbuch Verlag Berlin 1948.

66 Befehl Nr. 273 (Anm. 65), S. 13.

67 Befehl Nr. 273 (Anm. 65), S. 20.

68 Befehl Nr. 273 (Anm. 65), S. $16 f$.

69 Befehl Nr. 273 (Anm. 65), S. $18 f$.

70 Befehl Nr. 273 (Anm. 65), S. 18. 
3 Von den geschlossenen Fürsorgeheimen für Geschlechtskranke in der SBZ zu den geschlossenen Venerologischen Stationen in der DDR

mit Geschlechtskranken, Krankheitsverdächtigen und sogenannten HwGPersonen - unter anderem die Tendenz, Personen, die unter dem Verdacht standen geschlechtskrank zu sein, sofort zwangsweise zu hospitalisieren. Auf solche Traditionen wird sich in der DDR immer wieder bezogen. Sie begründen unter anderem die rechtswidrigen Einweisungen in den 196oer und 1970er Jahren in die geschlossene Venerologische Station in Leipzig-Thonberg.

\subsection{Die Umsetzung der SMAD-Befehle am Beispiel geschlossener Fürsorgeheime für Geschlechtskranke}

Die konkrete Umsetzung der drei SMAD-Befehle Nr. 25, 30 und 273 in der SBZ/ DDR lässt sich an verschiedenen Beispielen gut nachvollziehen - etwa hinsichtlich der Bekämpfung der Prostitution. ${ }^{71}$ Ein wichtiges Wirkungsfeld war die Einrichtung von geschlossenen Fürsorgeheimen für Prostituierte und Geschlechtskranke. Solche Häuser existierten in der gesamten SBZ/DDR, so zum Beispiel in Brandenburg, ${ }^{72}$ in Gräfentonna oder in Leipzig-Thonberg. ${ }^{73}$ Die Fürsorgeheime für Geschlechtskranke wiesen nicht nur untereinander Ähnlichkeiten auf, sondern waren an vielen Stellen eine Art Nukleus der in den 1950er und 196oer Jahren entstehenden geschlossenen Venerologischen Stationen. Dabei stand vor allem die Funktion der Fürsorgeheime paradigmatisch für die geschlossenen Venerologischen Stationen.

Für die konkrete Ausführung der einzelnen SMAD-Befehle wurden von den Landesverwaltungen Rundverfügungen erstellt. Laut zweier Rundverfügungen der Landesverwaltung Sachsen sollten im Frühjahr 1946 zur Durchführung des SMAD-Befehls Nr. 30 in den Städten Bautzen, Chemnitz, Dresden, Görlitz, Leipzig und Zwickau Fürsorgeheime eingerichtet werden. ${ }^{74}$ Die Einrichtung in Zwickau sollte in der Gefängnisanstalt Schloss Osterstein untergebracht werden. ${ }^{75}$ Schloss Osterstein, das 1292 erstmals urkundlich erwähnt wurde, war bereits 1775 in eine „Correktions- und Arbeitsanstalt“ für insgesamt 200 weibliche und männliche Häftlinge umgebaut worden. ${ }^{76}$ Zwischen März 1933 bis Februar 1934 war im Schloss Osterstein ein Konzentrationslager mit

71 Falck U (1998) VEB Bordell. Geschichte der Prostitution in der DDR. Ch. Links Verlag Berlin.

72 Pohl: Justiz in Brandenburg 1945-1955 (Anm. 45); Krumbiegel: Polikliniken in der SBZ, DDR (Anm. 45).

73 Korzilius: „Asoziale“ und „Parasiten“ im Recht der SBZ, DDR (Anm. 43), S. 99.

74 StAZ: R3/1032. Fürsorgeheim Schloß Osterstein: Rundverfügung 57. Maßnahmen zur Bekämpfung der Geschlechtskrankheiten. Landesverwaltung Sachsen - Gesundheitsweisen. Dresden, den 25. Februar 1946. Bl. 18v; StAZ: R3/1032. Fürsorgeheim Schloß Osterstein: Rundverfügung 64. Anordnung zur Bekämpfung der Geschlechtskrankheiten im Bundesland Sachsen der Landesverwaltung Sachsen. Landesverwaltung Sachsen Gesundheitsweisen. Dresden, den 4. März 1946. Bl. 20r.

75 StAZ: R3/1032. Fürsorgeheim Schloß Osterstein: Stadtgesundheitsamt vom 16. 3. 1946. Rundverfügung der Landesverwaltung Sachsen Nr. 64. Abs. 5, Fürsorgeheim für Prostituierte.

76 Baganz C (2005) Zwickau (Schloss Osterstein). In: Benz W, Distel B (Hrsg.) DER ORT DES TERRORS. Geschichte der nationalsozialistischen Konzentrationslager. Bd. 2, Frühe Lager, Dachau, Emslandlager. 227-230. Beck München. 
insgesamt 750 Häftlingen eingerichtet, anschließend diente das Schloss bis 1963 als Gefängnis. ${ }^{77}$ Nun sollte im linken Flügel des Gebäudes ein Fürsorgeheim für Geschlechtskranke eingerichtet werden. Hierzu wurde im Frühjahr 1946 mit dem Leiter vereinbart, dass vorerst 30 Betten aufgestellt werden konnten. Die Überwachung der Eingewiesenen sollte durch Angestellte bzw. Beamte der Gefängnisanstalt Zwickau erfolgen. Für die medizinische Betreuung war ein Facharzt für „Frauenkrankheiten“ vorgesehen. ${ }^{78}$ Bereits Anfang April 1946 wurde verbindlich erklärt, dass geschlechtskranke Personen ab sofort von den Gesundheitsämtern in das Zwickauer Fürsorgeheim eingewiesen werden konnten. ${ }^{79}$ Nach Interventionen durch Kommissionen der SMAD, die im Mai 1946 durch das Bundesland Sachsen reisten und die Einrichtungen prüften, wurde die Bettenzahl im Fürsorgeheim von 30 auf 300 erhöht. Nun konnte das Fürsorgeheim mit 250 weiblichen und 50 männlichen Personen belegt werden. ${ }^{80}$ Wie aus monatsweise geführten Beleglisten hervorgeht, die ab Oktober 1946 geführt werden mussten, waren seitdem durchschnittlich 130 bis 150 der 300 Betten belegt. ${ }^{81}$

Im November 1946 wurde eine „Hausordnung für das Fürsorgeheim“ “82 beschlossen, in welcher ihre Struktur und Funktion bestimmt wurde: Einrichtung des Fürsorgeheims auf Grundlage des SMAD-Befehls Nr. 30 ( $\$ 1)$, 250 Betten für Frauen und 50 Betten für Männer ( $\$ 2$ ), die Trennung in eine Frauenund Männerabteilung mit je einer Lues- und einer Gonorrhoeabteilung ( $\$ 7$ ). Die Funktion des Fürsorgeheims stand in $\mathbb{s} 3$ : „Das Fürsorgeheim ist 1. Behandlungsstätte, 2. Isolierstätte, 3. Straf- und Arbeitsanstalt und 4. Umerziehungsheim. “ Darüber hinaus wurde der Tagesablauf in $\mathbb{1}$ 1o geregelt: „Im Fürsorgeheim wird eine 8-stündige Arbeitszeit durchgeführt. Die Fürsorge-Insassen arbeiten in der Zeit von $8 \mathrm{Uhr}$ morgens bis $6 \mathrm{Uhr}$ abends mit einer zweistündigen Mittagspause. (...)Tragen von Schmuck ist nicht gestattet. Geld und Schmuckgegenstände sind bei der Aufnahme von dem Polizeiorgan des Fürsorgeheims abzunehmen. "Der $\$ 12$ installierte Aufseherinnen aus den Reihen der Zwangseingewiesenen: „In den Sälen wird durch die Heimleiterin nach Rücksprache mit dem ärztlichen Leiter je eine Aufsichtsperson aus den Reihen der Fürsorgeheim-Insassen bestimmt. " Die Aufgaben dieser Aufsichtsperson waren im $\$ 13$ niedergelegt: „In den Arbeitssälen sind Privatgespräche während der Arbeitszeit untersagt. Hier hat Ordnung, Ruhe und Disziplin zu herrschen.

77 Baganz: Zwickau (Schloss Osterstein) (Anm. 76), S. 228.

78 StAZ: R3/1032. Fürsorgeheim Schloß Osterstein: Stadtgesundheitsamt vom 16. 3. 1946.

79 StAZ: R3/1032. Fürsorgeheim Schloß Osterstein: Stadtgesundheitsamt 6. 4. 46, An das Stadtgesundheitsamt Plauen, Einweisung in das Fürsorgeheim Zwickau. Bl. 12.

80 StAZ: R3/1032. Fürsorgeheim Schloß Osterstein: Rat der Stadt Zwickau Sachsen - Gesundheitsamt. Zwickau, den 17. Juni 1946. Bl. 18.

81 StAZ: R3/1032. Fürsorgeheim Schloß Osterstein: Rat der Stadt Zwickau (Sachsen). An das Gesundheitsamt Zwickau. Betr.: Monatsbericht an die Landesverwaltung. Zwickau, den 26. Oktober 1946. Bl. 31, Bl. 36f.

82 StAZ: R3/1032. Fürsorgeheim Schloß Osterstein: Hausordnung für das Fürsorgeheim. Zwickau, den 9. November 1946. 
3 Von den geschlossenen Fürsorgeheimen für Geschlechtskranke in der SBZ zu den geschlossenen Venerologischen Stationen in der DDR

Verantwortlich hierfür ist die Heimleitung und die Aufsichtsperson." Auch Sanktionen bzw. einzelne Strafen wurden unter anderem in den $\mathbb{s} 14$ und 15 der Hausordnung aufgeführt: „Solange die erforderliche Disziplin und Ordnung im Fürsorgeheim nicht vorhanden ist, ist jede Paketannahme verboten. “ Die Erziehung der Zwangseingewiesenen sollte nicht nur über die tägliche Arbeit oder durch das straffe Zeitregiment, sondern über pädagogische Unterweisungen gewährleistet werden, wie aus dem $\mathbb{1} 18$ hervorgeht: „Um der Aufgabe der Umerziehung der Fürsorgeheim-Insassen gerecht zu werden, hat die Heimleitung zusammen mit dem ärztlichen Leiter dafür zu sorgen, dass genügend Kräfte aus den Frauenausschüssen, aus der Lehrerschaft und aus dem Personal der Fürsorgerinnen herangezogen werden, die eine Schulung unter den Fürsorgeheim-Insassen durchführen. " ${ }_{3}$

83 StAZ: R3/1032. Fürsorgeheim Schloß Osterstein: Hausordnung für das Fürsorgeheim (Anm. 82), Bl. 33-35. 
3.2 Die Umsetzung der SMAD-Befehle am Beispiel geschlossener Fürsorgeheime für Geschlechtskranke

Der Rat der Stadt

Zwickau/Sachsen

- Gesundheirsamt -

\section{Hausordnung für das Furssorgeheim}

1. Des Fursorgeheim ist auf Grund des Befehls 030 der SMA von der Stadtverwaltung Zwi okal eingeriehtet woxden und im Zuchthaus, Katharinenic r ch of 12, untergebracht.

2. Im Füsorgeheim stehen 250 Betten fur Frauen und 50 Betten fur Mäner bereit.

3. Das Tirsorgehein ist
1. Behandiungsstiltte,
2. Isollerstätte,
3. Straf- und Arbeltsanstalt und
4. Umerziehungshein.

AIle Aufgaben stehen zueinander in gleichen Verhältnis.

4. Das Fursorgeheim 1st dem Gesundheitsant angegliedert.

5. DLe Gesamtleitung des Fursorgehelras liegt in den Händen des ärztlichen Leiters.

6. Die Verwaltung des Zuchthauses stellt eine Aufsiohtsperson fur das rixsorgehein, die die Belange des Zuehthauses im F. F. vertritt. Diese hat innerhazb des Fursorgehel rs oie polizeigewalt.

7. Das riraorgeheim hat eine Mllinner-und eine rrauenabteilung mit je einer Abtelilung flir Lues-und fïr Gonorrhoekranke. Fir die inzelnen Abteilungen steht ein besonderex Arbeits- und Schlafsaal mur Verfügung.

8. Das Sehwesternpersonal ubernimat die pflegerisehen und kilnischen Arbeiten und die Fihrung eines Teiles der Krankengeschichten, dag Büropersonal die schriftlichen, rechnerischen und verwaltungsmabigen Arbeiten. Dle Fürsorge ibernimnt die Heimleiterin (Arbeitsbeschaffung, Arbeitsausgabe und Arbeitselnteilung). Der arztliche leiter hat noben den klinischen Arbeiten die Aufsioht uber das gesante Personal und uber die Arbeit in Firsorgeheim. Bei polizeilichen Ma Bnahmen ausserhalb des Fursorgeheims muss das Personal die Ortspolizelbehörde baw. (0. $m .46 !$ die Bahnhof smisesion in Anspruch nehmen.

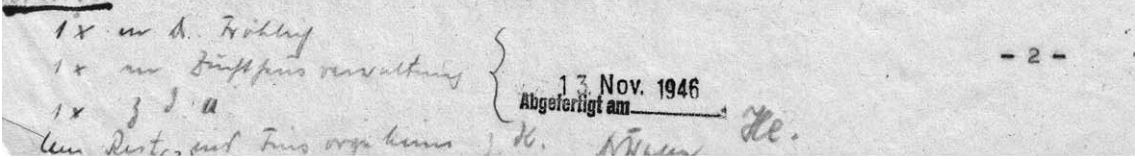

Abb. 3 „Hausordnung für das Fürsorgeheim“ 
3 Von den geschlossenen Fürsorgeheimen für Geschlechtskranke in der SBZ zu den geschlossenen Venerologischen Stationen in der DDR

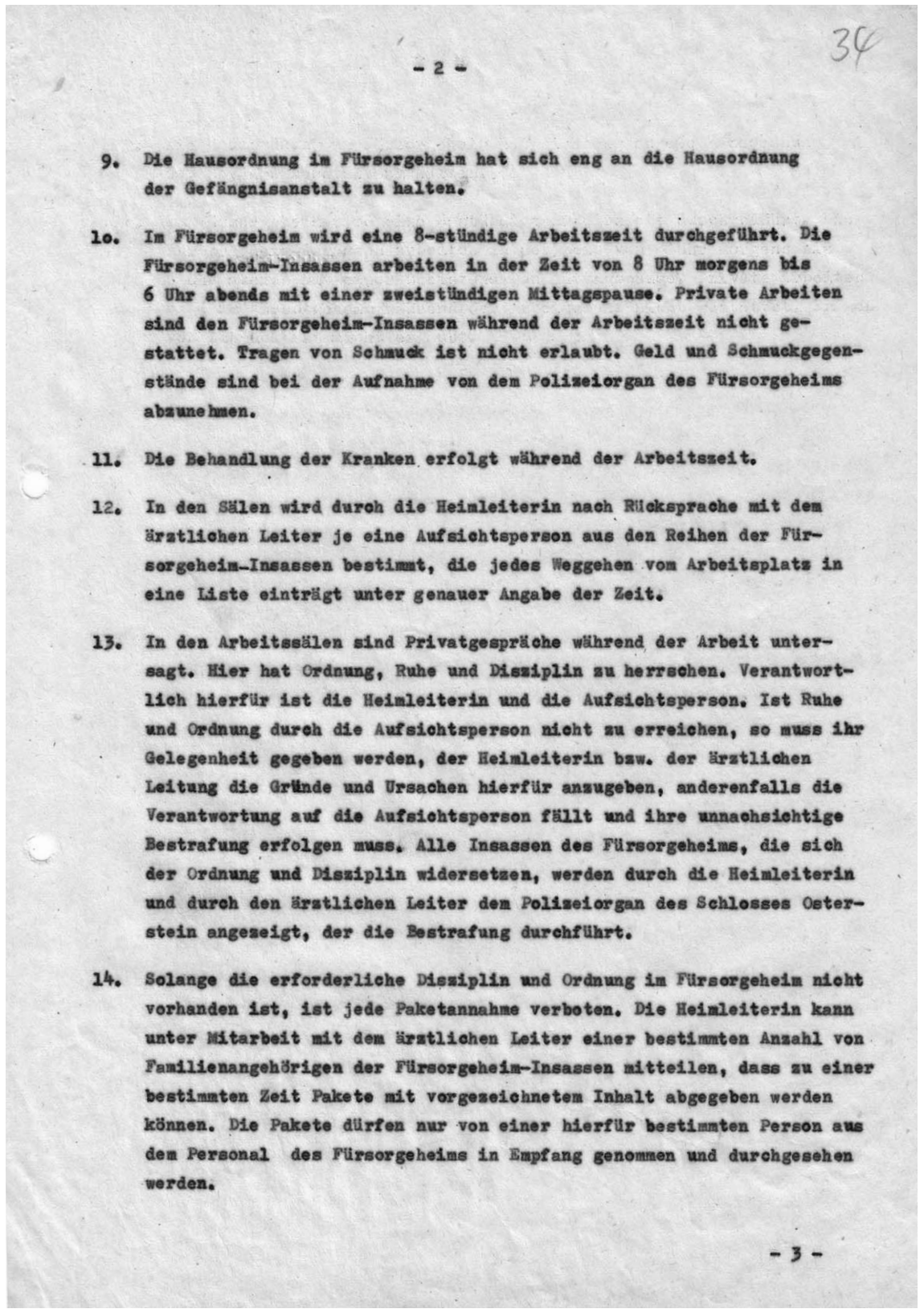

Fortsetzung von Abb. 3 „Hausordnung für das Fürsorgeheim“ 
3.2 Die Umsetzung der SMAD-Befehle am Beispiel geschlossener Fürsorgeheime für Geschlechtskranke

15. Auch das Absenden von Karten und Briefen an dLle Angehörigen der Fursorgehelm-Insassen ist sunächst nioht gestattet. D1e Heinleiterin kann, sobald sie die GewiBheit hat, dass Ordnung und Disziplin in Fursorgehein herrschen, einer bestimmten Anzahl von Insassen die Molglichkeit geben, Innerhalb einer bestimnten Frist an Ihre Familienangehörigen zu schreiben. Die Heimleiterin ist verprilchtet, jeden Brief und jede Karte genauestens durchsusehen und die durchgesehene Post den Pollselorgan au ubergeben.

16. Das Fiusorgehein daxi nie ohne Aufaleht seitens des Personals gelassen werden. Es muss daher ein Sonnabend- und Sonntagsdienat eingerichtet werden. Das Personal hat sich in diesen Sonnabend- und Sonntagsdienst ou teilen. Sachgemässe Anordniung trifft in dieser Angelegenheit der äratliche Leiter. Dem Personal ist es in der folgenden Woche gestattet, nach dem Sonntagsdienst einen freien Tag wu nehmen. Auch am Sonntag hat in Fursorgeheim Ordnung und Disaiplin an herrschen.

17. Es Lst Aufgabe der Heimleiterin und des äratliehen Leiters, an den Sonntagen fü eine sachgemässe Beschüftigung baw. für eine Abwechslung oder fur Iralehung durch Vorträge und Unterricht Sorge au tragen.

18. Un der Aufgabe der Unerziehung der Firsorgeheim-Insassen gerecht au werden, hat die Heinleiterin zusammen mit den äratlichen Leiter dafir zu sorgen, dass genilgend Krafte aus den Fravenauschlssen, aus der Lehrersehaft und aus dere Personal def Fursorgerinnen herangesogen werden, die eine Sehulung unter den Fursorgeheim-Insassen durchfuhren.

19. Bntstehen unter dem Personal des Fursorgeheims Schwierigkeiten oder Unklaxheiten, so haben sich die betreffenden Personen sunk̈chst an den arstlichen Ledter au wenden. Wenn Abhlife durch diesen nicht erfolgt, steht es jeden frei, schriftlioh bein Amtsarat die Kagen voraubringen.

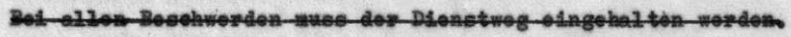

20. Das Polizelorgan in Fursorgeheim kann aur Erledigung der Hausarbeiten in Firsorgehein von der Helmleiterin Peraonal anfordern. Die Heimleiterin stellt das Personal nach Rucksprache wit den äratilehen lelter rechtzestig und in ausreichender Zahl zur Verfigung. 
3 Von den geschlossenen Fürsorgeheimen für Geschlechtskranke in der SBZ zu den geschlossenen Venerologischen Stationen in der DDR

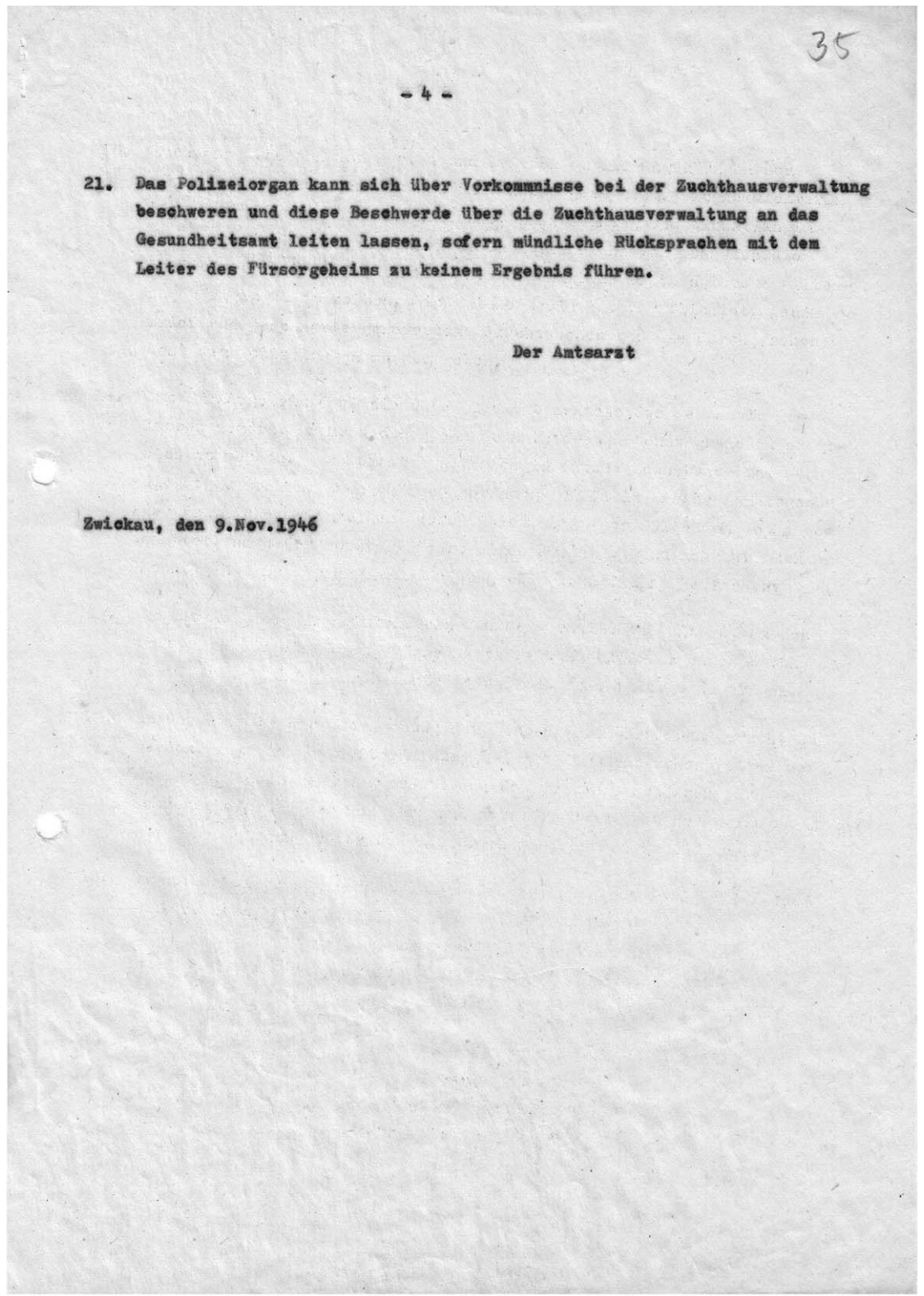

Fortsetzung von Abb. 3 „Hausordnung für das Fürsorgeheim“ 
Dieser Mikrokosmos aus Ordnung, Disziplin und Erziehung wurde ständig überwacht. So ließ unter anderem die verantwortliche Gefängniswachtmeisterin Frau Wadischat das medizinisch-pflegerische Personal und die Zwangseingewiesenen regelmäßig bespitzeln. ${ }^{84}$ Dafür bestimmte sie aus der Gruppe der Patienten „sog. Kalfaktorinnen“. ${ }^{85}$ Cleichzeitig herrschte im Fürsorgeheim Zwickau Mangel an fast allem. Es fehlte an Bettwäsche, Decken, Nahrungssowie an Genussmitteln. ${ }^{86}$ Die Pflegerinnen stahlen Brot von den Zwangseingewiesenen, und diese wiederum nahmen sich untereinander die geringen Brotrationen weg. Zigaretten wurden für 6 Reichsmark pro Stück gehandelt. ${ }^{87}$ Besonders gravierend wirkte sich der Mangel an Bettwäsche und Decken im Winter 1946/47 aus. Die Patienten mussten in Räumen schlafen, „in denen nachts eine Temperatur von minus 8 Grad herrschte. " 88

Bereits im Februar 1946 zeichnete sich das Ende des Fürsorgeheims in Zwickau ab, denn die dortigen Verhältnisse waren aus Sicht des Amtsarztes vor allem bei den Männern untragbar: „In einem engen, niedrigen Raum sind hier die Go.- und Lueskranken zusammen eingepfercht. Die Gefahr der gegenseitigen Ansteckung ist hier eine besonders große. Die Verhältnisse in der männlichen Abteilung des Fürsorgeheimes sind für jedes menschliche Gefühl unhaltbar." ${ }^{89}$ Mit der beschriebenen Situation in der Männerabteilung lag ein klarer Verstoß gegen den SMAD-Befehl Nr. 30 und die entsprechenden Rundverfügungen vor, in denen explizit eine Trennung der Zwangseingewiesenen nach Geschlecht und Krankheit gefordert war. Da sich die Leitung des Zuchthauses weigerte, weitere Räume für die getrennte Unterbringung der männlichen Patienten zur Verfügung zu stellen, wurde das Fürsorgeheim im Juni 1947 aufgelöst bzw. nach Crimmitschau verlegt. ${ }^{\circ}$

Die nur knapp einjährige Geschichte des Fürsorgeheims in Zwickau steht dennoch symptomatisch für andere Fürsorgeheime, aus denen in den 1950er Jahren geschlossene Venerologische Stationen wurden. So glichen die Zwickauer Bedingungen denen in Leipzig-Thonberg. Auch dort war die Unterbringung im Winter 1946/47 mangelhaft, es gab kaum Decken, und die Räume hatten teilweise eine Höchsttemperatur von 6 Grad Celsius. ${ }^{91}$ Dennoch wurde LeipzigThonberg nicht geschlossen, sondern bis 1952 betrieben. Gerade dieser Aspekt zeigt, dass das Fürsorgeheim in Zwickau nicht aufgrund einer allgemein un-

84 StAZ: R3/1032. Fürsorgeheim Schloß Osterstein: Protokoll über eine Dienstbesprechung im Fürsorgeheim am 11.1.1947. Zwickau, den 17. Januar 1947. Bl. 40v.

85 StAZ: R3/1032. Fürsorgeheim Schloß Osterstein: Protokoll über eine Dienstbesprechung (Anm. 84), Bl. 40r.

86 StAZ: R3/1032. Fürsorgeheim Schloß Osterstein: Der Leiter der Vollzugsanstalten Zwickau. Zwickau, den 7. September 1946. Bl. 26.

87 StAZ: R3/1032. Fürsorgeheim Schloß Osterstein: Protokoll über eine Dienstbesprechung (Anm. 84), Bl. 40r.

88 StAZ: R3/1032. Fürsorgeheim Schloß Osterstein: An den Rat der Stadt. Zwickau, den 24. Februar 1947. Bl. 44v.

89 StAZ: R3/1032. Fürsorgeheim Schloß Osterstein: An den Rat der Stadt (Anm. 88). Bl. 44v.

90 StAZ: R3/1032. Fürsorgeheim Schloß Osterstein: An den Rat der Stadt Zwickau. Betr.: Auflösung des Fürsorgeheims. Zwickau, den 19. Juni 1947. Bl. 55.

91 Korzilius: „Asoziale“ und „Parasiten“ im Recht der SBZ, DDR (Anm. 43), S. 98. 
3 Von den geschlossenen Fürsorgeheimen für Geschlechtskranke in der SBZ zu den geschlossenen Venerologischen Stationen in der DDR

zureichenden Ausstattung aufgelöst wurde, sondern weil die männlichen Geschlechtskranken nicht nach Art der Infektion getrennt werden konnten. Der Raummangel, der einen Verstoß gegen die SMAD-Befehle Nr. 25 und 30 darstellte, führte letztlich zur Schließung des Fürsorgeheims am Standort Zwickau. Die Kontrollkommissionen der SMAD setzten ihre Befehle gegenüber den Institutionen aber auch gegenüber den Zwangseingewiesenen dort rigoros durch. Abweichungen wurden sanktioniert und normiert.

Darüber hinaus findet sich in den Fürsorgeheimen eine Art institutioneller Ursprung der geschlossenen Venerologischen Stationen. So geht unter anderem aus dem Fürsorgeheim für Geschlechtskranke Leipzig-Thonberg im Jahr 1952 die geschlossene Venerologische Station Leipzig-Thonberg hervor. ${ }^{92}$ Aber auch die Funktionsbestimmung der Heime - Behandlungsstätte, Isolierstätte, Straf- und Arbeitsanstalt und Umerziehungsheim - nehmen die Funktion der geschlossenen Venerologischen Stationen vorweg. Auch in der geschlossenen Venerologischen Station in Halle (Saale) wurden die Zwangseingewiesenen behandelt, isoliert, erzogen und zur „Arbeitstherapie“ verpflichtet. Es ging bei beiden Institutionen um die Behandlung von geschlechtskranken Personen sowie deren Isolierung und Erziehung, die wiederum vergleichbar ist. Denn sowohl in Fürsorgeheimen als auch in geschlossenen Venerologischen Stationen verlief die Erziehung über die Ausübung der täglichen Arbeit. Diese war zeitlich genau getaktet und strukturierte den Tagesablauf der Zwangseingewiesenen und des medizinisch-pflegerischen Personals. Des Weiteren ist die Instanz der Kalfaktorin im Fürsorgeheim Zwickau wegweisend. Auch in den geschlossenen Venerologischen Stationen werden Stubenälteste eine ähnliche Funktion haben wie die Kalfaktorinnen im Fürsorgeheim, sodass sich die damit verbundene Überwachung und Denunziation später in ähnlicher Form wiederfindet.

\subsection{Die Umsetzung der SMAD-Befehle und die medizinische Versorgung von Geschlechtskranken in der SBZ und der DDR}

Nach dem SMAD-Befehl Nr. 30 waren die Pflichtkurse zur Aus- und Weiterbildung ein fester Bestandteil der Maßnahmen zur Bekämpfung von Geschlechtskrankheiten. In $\mathbb{1} 10$ war festgelegt, dass in den Kliniken für Hautund Geschlechtskrankheiten der Städte Berlin, Dresden, Greifswald, Halle (Saale), Leipzig und Rostock eine Anlernung von Ärzten für Geschlechtskrankheiten aus der Zahl der allgemeinen Ärzte in sechswöchigen Kursen vorzunehmen sei. ${ }^{93}$ Ziel dieser Kurse war es, genaue Kenntnisse über die Erkennung von Geschlechtskrankheiten zu vermitteln und die Ärzte in die Lage zu versetzen, in eigener Verantwortlichkeit Geschlechtskrankheiten zu behandeln.

92 Korzilius: „Asoziale“ und „Parasiten“ im Recht der SBZ, DDR (Anm. 43), S. 99.

93 StAZ: R3/1032. Fürsorgeheim Schloß Osterstein: Befehl des Obersten Chefs (Anm. 64), Bl. 6. 
Die Inhalte, die während der Pflichtkurse vermittelt wurden, lassen sich aus dem „Bericht über den 6. Pflichtkursus zur Bekämpfung der Geschlechtskrankheiten in der Universitäts-Hautklinik zu Leipzig (vom 28. X. bis 7. XII. 1946)“ ableiten. ${ }^{94}$ Die Analyse dieses Berichts zeigt nicht nur den seinerzeit aktuellen Stand des medizinischen Wissens und verdeutlicht damit die therapeutische Praxis. Vielmehr werden die medizinischen Grundlagen deutlich, die bis weit in die 196oer Jahre die Praxis prägten. Dies trifft vor allem auf die sogenannten Fieberspritzen zu, die als Provokationsmaßnahme bis in die 196oer Jahre in den geschlossenen Venerologischen Stationen Anwendung fanden. Aber auch die sogenannten Fiebertherapien waren ein Mittel, um noch 20 Jahre später Gonorrhoe zu behandeln.

Der hier vorgestellte Bericht über einen Pflichtkurs gibt Einblicke in die venerologische Theorie und Praxis an der Hautklinik der Universität Leipzig. Dort wurden die Pflichtkurse durchgeführt, und dort war seit 1947 auch Wolfgang Johannes Gertler (1904-1982) tätig. Gertler hatte nach seinem Abitur 1924 in Chemnitz zunächst in Freiburg Philosophie und Musikwissenschaft studiert. 95 Nach dem Studienabschluss wurde er 1929 mit einer Dissertation über die frühen Klavierwerke Robert Schumanns zum Doktor der Philosophie promoviert. ${ }^{96}$ Anschließend ging Gertler nach Berlin, um ein Studium der Humanmedizin aufzunehmen. 1936 wurde Gertler mit der Arbeit Über die Wirkung der stereoisomeren Modifikationen des Tripeptids Leucylglycyl-glycin auf die Spaltung der Pankreas- und Leberesterase ${ }^{97}$ zum Doktor der Medizin promoviert. Es folgten 1937 die dermatologische Fachausbildung in Berlin sowie die Fortsetzung der Facharztausbildung bei Heinrich Gottron (1890-1974) in Breslau. 1945 floh Gertler aus Breslau und übernahm eine Facharztpraxis in Jena. 1947 habilitierte er sich für Dermatologie an der Universität Jena mit der unveröffentlichten Arbeit Experimentelle Untersuchungen über den Sulfonamidgehalt in Körperflüssigkeiten bei Gonorrhoebehandlung. 1947 wechselte Gertler an die Hautklinik der Universität Leipzig, die von Karl Linser (1895-1979) geleitet wurde. Dort wurde er zum Oberarzt und stellvertretenden Klinikleiter ernannt. 1950 erhielt er eine Professur und wurde 1951 Direktor der Hautklinik. 1962 trat er die Nachfolge von Linser an und wurde auf das Ordinariat für Dermatologie an der Charité berufen..$^{98}$

94 Von Begg-Albensberg H-H (1946) Bericht über den 6. Pflichtkursus zur Bekämpfung der Geschlechtskrankheiten in der Universitäts-Hautklinik zu Leipzig (vom 28. X. bis 7. XII. 1946). Markkleeberg, S. II.

95 Schreier I (1985) Die Entwicklung der Dermato-Venerologie an der Medizinischen Fakultät der Universität Leipzig vom Anfang des 19. Jahrhunderts bis zum Beginn der sozialistischen Umgestaltung an der Universität 1951. Med. Diss., Leipzig, S. 50.

96 Gertler W (1929) Robert Schumann in seinen frühen Klavierwerken. Phil. Diss., Freiburg im Breisgau.

97 Gertler W (1936) Über die Wirkung der stereoisomeren Modifikationen des Tripeptids Leucylglycyl-glycin auf die Spaltung der Pankreas- und Leberesterase. Med. Diss., Berlin.

98 Schreier: Die Entwicklung der Dermato-Venerologie (Anm. 95). 
3 Von den geschlossenen Fürsorgeheimen für Geschlechtskranke in der SBZ zu den geschlossenen Venerologischen Stationen in der DDR

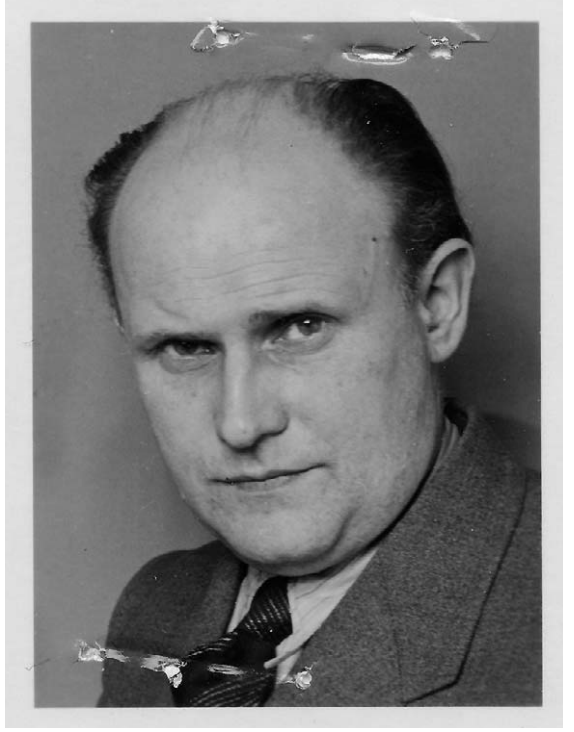

Abb. 4 Porträt von Wolfgang Gertler (1904-1982)

Der Pflichtkurs an der Hautklinik der Universität Leipzig gestaltete sich wie folgt: Sämtliche Kursteilnehmer mussten gruppenweise und im achttägigen Wechsel folgende Stationen und Abteilungen durchlaufen: 1. Männerstation A, 2. Männerstation B, 3. Frauenstation, 4. Poliklinik für Männer, 5. Poliklinik für Frauen, 6. Laboratorien oder wahlweise erneut eine der bereits genannten Stationen. Zusätzlich wurden Vorlesungen und Praktika besucht, um die Gelegenheit zu praktischer und poliklinischer Tätigkeit zu erhalten. ${ }^{99}$ In dem Pflichtkurs wurden die Ärzte über die verschiedenen Arten der Geschlechtskrankheiten, ihre Formen, ihre jeweiligen Erscheinungsbilder und die Diagnosen informiert - beispielsweise Gonorrhoe, Gonorrhoe posterior bei männlichen und weiblichen Patienten sowie bei Kindern. ${ }^{100}$ Des Weiteren wurden Therapieformen im Allgemeinen und in jeweils spezifischer Form vorgestellt etwa die Therapie der Gonorrhoe mit 1. Sulfonamiden, 2. Fiebertherapie und 3. Penicillin bei männlichen und weiblichen Patienten sowie bei der kindlichen Gonorrhoe. ${ }^{101}$ Schließlich wurde auf die Nebenwirkungen der einzelnen Therapien, die Frühzeichen und deren Vorbeugung hingewiesen - zum Beispiel Salvarsanschäden der Nieren. ${ }^{102}$ Darüber hinaus wurden Fragen des labortechnischen Nachweises von Geschlechtskrankheiten geklärt - etwa zum Nachweis der Ducreyschen Bazillen bei einem Ulcus molle (weicher Schanker). ${ }^{103}$

99 Von Begg-Albensberg: Bericht über den 6. Pflichtkursus (Anm. 94), S. III

100 Von Begg-Albensberg: Bericht über den 6. Pflichtkursus (Anm. 94), S. IV-VI.

101 Von Begg-Albensberg: Bericht über den 6. Pflichtkursus (Anm. 94), S. VI-VIII.

102 Von Begg-Albensberg: Bericht über den 6. Pflichtkursus (Anm. 94), S. XVIII-XXII.

103 Von Begg-Albensberg: Bericht über den 6. Pflichtkursus (Anm. 94), S. XXIV. 
In dem Bericht findet sich auch eine Beschreibung, wie die Untersuchung venerisch erkrankter Personen stattfinden sollte: „Alle Personen, die auf Geschlechtskrankheiten untersucht werden sollen, haben sich vor Beginn der ärztlichen Untersuchung völlig zu entkleiden!“ Hierzu waren Umkleidekabinen oder Vorhänge zur Abtrennung von einzelnen Räumen nötig. „Die Untersuchung (...) beginnt mit der Inspektion und allgemeinen Untersuchung aller Körperregionen." Hierfür wäre es zweckmäßig, einem Schema zu folgen: 1. Kopf, Gesicht, Mundhöhle, Rachen, 2. Hals, 3. obere Extremitäten, 4. Stamm, 5. untere Extremitäten, 6. Analgegend und 7. Genitalstatus. „Nach dieser generellen Untersuchung muss die Erhebung des genauen Genitalstatus in sachkundiger und umfassender Weise angeschlossen werden. " ${ }^{104}$

Bei der Behandlung der Gonorrhoe wurden drei Varianten angegeben: Bei der Therapie mit Sulfonamiden sollte ein Stoß von 70 Gramm in fünf Tagen verabreicht werden. Als Nebenwirkung könnten schwere Nierenschäden auftreten. Der Heilungserfolg betrug 50\% und konnte durch eine Kombination mit Fiebertherapie auf 70\% erhöht werden. Bei der Fiebertherapie provozierte man gleichmäßige Fieberzacken. Dazu wurden wahlweise Olobinthin (40\% Terpentinöl) oder Turointol (40-60\% Terpentinöl) intramuskulär injiziert. Möglich war auch die intramuskuläre Injektion von Milch. Die Gabe von Milch verursache zwar Abszesse, die aber an sich nicht schädlich seien. Als dritte Therapie wurde die Penicillin-Behandlung vorgestellt. Dafür benötigte man 100.000 bis 200.00o Oxford-Einheiten. Alle zwei Stunden wurden 20.ooo Oxford-Einheiten intravenös verabreicht, was nach 20 Stunden die notwendige Dosis von 200.ooo Oxford-Einheiten ergab. ${ }^{105}$

Explizit empfiehlt der Bericht die Anwendung von Penicillin bei der Therapie von Geschlechtskrankheiten: „Das Penicillin brachte in der Behandlung der Gonorrhoe einen bedeutsamen therapeutischen Fortschritt. Die Krankheit hat ihren Schrecken für die Menschheit weitgehendst verloren. Kann doch die Go an einem Tag geheilt werden. Die Therapie der Syphilis soll nach den äusserst vorsichtig aufzufassenden Berichten in 8 Tagen (250.ooo O.E.) beendet sein. Die Zukunft wird zeigen, ob diese Behandlung vollständig ist. "Alternativ dazu wurde die Sulfonamidtherapie als bekanntes und erprobtes Mittel gegen Geschlechtskrankheiten dargestellt, bei dem ausdrücklich vor den Nebenwirkungen gewarnt wurde. ${ }^{106}$

Um den Erfolg der Therapie zu prüfen, waren drei Provokationen vorgeschrieben und der Nachweis von 15 negativen Abstrichen. Es gab die chemische, die mechanische und die biologische Provokation. Bei der chemischen Provokation wurde Lugolsche Lösung, eine Iod-Kaliumiodid-Lösung, in die Harnröhre eingeführt. Die mechanische Provokation bestand vor allem aus Massage. Bei der

104 Von Begg-Albensberg: Bericht über den 6. Pflichtkursus (Anm. 94), S. XXX, Hervorhebungen im Original.

105 Von Begg-Albensberg: Bericht über den 6. Pflichtkursus (Anm. 94), S. VII.

106 Von Begg-Albensberg: Bericht über den 6. Pflichtkursus (Anm. 94), S. XXIII. 
3 Von den geschlossenen Fürsorgeheimen für Geschlechtskranke in der SBZ zu den geschlossenen Venerologischen Stationen in der DDR

biologischen Provokation kamen Gonokokkenvakzine zum Einsatz. War die Therapie erfolgreich, folgten weitere acht Wochen der Nachbeobachtung. In diesen acht Wochen sollten weitere drei Provokationen durchgeführt werden. ${ }^{107}$

Wie neu die Behandlung mit Penicillin war, zeigen die unterschiedlichen Empfehlungen und Vorgaben zur Dosierung und Anwendung des Medikaments, die in der SBZ und in den ersten Jahren der DDR herausgegeben wurden. ${ }^{108}$ Unterschiede finden sich sowohl in der Dosierung als auch im Zeitraum der Anwendung. In Sachsen wurden in den Pflichtkursen zur Gonorrhoetherapie 200.ooo Oxford-Einheiten empfohlen, die alle zwei Stunden intravenös verabreicht werden sollten. ${ }^{109}$ Hingegen wurden in Berlin einmalig 300.000 OxfordEinheiten (öliges Depotpenicillin), einmalig 400.ooo Oxford-Einheiten (wässriges Depotpenicillin) oder vier Mal 50.000 Oxford-Einheiten in je dreistündigen Abständen vorgeschlagen. ${ }^{110}$ Diese unterschiedlichen Vorgaben wurden bis weit in die 1950er Jahre stark diskutiert. ${ }^{11}$ Dabei drehten sich die Auseinandersetzungen um die Form des verabreichten Penicillins - in wässriger Form oder als Depot - sowie um die Art der Behandlung - stationär oder ambulant. ${ }^{12}$ Die Debatte mündete 1957 in dem Bericht Neuere Ergebnisse auf dem Gebiet der praktischen Dermatologie, der von Wolfgang Gertler, in dieser Zeit Direktor der Dermatologischen Klinik der Karl-Marx-Universität Leipzig, herausgegeben wurde. ${ }^{{ }^{13}}$ Darin setzten sich Venerologen der gesamten DDR vor allem mit der Behandlung von Geschlechtskrankheiten auseinander. Die Penicillintherapie hatte sich Ende der 1950er Jahre durchgesetzt. Diskutiert wurden das vermehrte Auftreten von Rezidiven sowie die Anzahl der Provokationen zum Nachweis des Therapieerfolgs. Während einige Venerologen auf die Provokationen vollkommen verzichten wollten, setzen sich vor allem diejenigen Venerologen für die Beibehaltung der Provokationen ein, die geschlossene Venerologische Stationen betreuten. So plädierte unter anderem Dr. med. Heinz Hering (1917-1998), seit 1950 Direktor der Hautklinik Dresden-Friedrichstadt und verantwortlich für die geschlossenen Venerologischen Stationen für Männer und Frauen, für eine zeitlich gedehnte Hospitalisierung der HwG-Personen, um hinreichend viele Provokationen durchführen zu können. ${ }^{114}$ Provokationen wurden auch noch in den 197oer Jah-

107 Von Begg-Albensberg: Bericht über den 6. Pflichtkursus (Anm. 94), S. VIII.

108 BArch Best. DQ 1. Nr. 4228, unpag. Empfehlungen zur Behandlung von Geschlechtskranken.

109 Von Begg-Albensberg: Bericht über den 6. Pflichtkursus (Anm. 94), S. VII.

110 LA Berlin: C Rep. 118 - Magistrat von Berlin, Abteilung Gesundheits- und Sozialwesen. Nr. 863: Verordnung zur Bekämpfung der Geschlechtskrankheiten 1955-1956. Vorschläge für Richtlinien zur ambulanten Behandlung der Geschlechtskrankheiten, S. 2.

111 Vgl. hier etwa: LA Berlin: C Rep. 118 - Magistrat von Berlin, Abteilung Gesundheits- und Sozialwesen. Nr. 863: Verordnung zur Bekämpfung der Geschlechtskrankheiten 1955-1956. Schreiben Linsers an den Magistrat von Berlin.

112 Gertler W (1956) Zur Penicillinbehandlung der Syphilis. Das deutsche Gesundheitswesen. Zeitschrift für Medizin XI, 977-987.

113 Gertler W (1957) Neuere Ergebnisse auf dem Gebiet der praktischen Dermatologie. VEB Verlag Volk und Gesundheit Berlin.

114 Hering H (1957) Aktuelle Fragen auf dem Gebiet der Gonorrhoe. In: Gertler W (Hrsg.) Neuere Ergebnisse auf dem Gebiet der praktischen Dermatologie. 180-191. VEB Verlag Volk und Gesundheit Berlin. 
3.4 Die „Verordnung zur Verhütung und Bekämpfung von Geschlechtskrankheiten“ vom 23. Februar 1961

ren eingesetzt und galten als Standard zur Prüfung eines Therapieerfolgs, wie unter anderem in Gertlers Systematischer Dermatologie beschrieben wird. ${ }^{115}$

Ziel der im SMAD-Befehl Nr. 30 vorgeschriebenen Ausbildung war eine einheitliche Behandlung der Geschlechtskrankheiten unter dem Primat der öffentlichen Behandlungsstellen. Alle Ärzte, die künftig in der Venerologie tätig waren, auch die Fachärzte, sollten den Anforderungen der modernen Diagnostik und Therapie von Geschlechtskrankheiten gewachsen sein. ${ }^{116}$ Dazu gehörte die Behandlung mit dem modernen Penicillin, das die Gonorrhoe innerhalb von 24 Stunden und die Syphilis innerhalb von 8 Tagen ausheilte. Die Venerologen in den Fürsorgeheimen und geschlossenen Venerologischen Stationen waren durch die konsequenten Pflichtkurse alle auf einem ähnlich aktuellen Wissensstand. Der Einsatz von Penicillin hätte bei ambulanter Anschlussversorgung ( 3 bis 4 Provokationen und 15 Abstriche) die Fürsorgeheime und geschlossenen Stationen verzichtbar gemacht, was dazu führt, dass die weiteren Funktionen der Fürsorgeheime und geschlossenen Venerologischen Stationen - Isolation, Arbeitsdienst und Erziehung - in den Vordergrund treten. Diese drei Funktionen wurden in den Fürsorgeheimen und teilweise in den Venerologischen Stationen durch die Ausgangssperre, die Überwachung, das strenge Zeitregime, die tägliche Arbeit und die pädagogischen Unterweisungen realisiert. Somit prägten Isolation, Arbeitsdienst und Erziehung den Alltag in den Einrichtungen, und der Aspekt der ärztlichen Versorgung wurde vernachlässigt.

\subsection{Die „Verordnung zur Verhütung und Bekämpfung von Geschlechtskrankheiten“ vom 23. Februar 1961}

Karl Linser hatte in seiner Funktion als Präsident der Deutschen Zentralverwaltung für das Gesundheitswesen in der SBZ (1947-1948) das Vorwort zur Broschüre Die gesetzlichen Bestimmungen zur Bekämpfung der Geschlechtskrankheiten in der sowjetischen Besatzungszone Deutschlands geschrieben. ${ }^{117}$ Hier lobte der Dermatologe die Bestimmungen des SMAD-Befehls Nr. 273 und verteidigte die rigiden Maßnahmen der Zwangshospitalisierung. Knapp zehn Jahre später ging von Linser eine Initiative zur gesetzlichen Neuregelung der Bekämpfung von Geschlechtskrankheiten aus. Zu dieser Zeit war er Professor für Dermatologie und Venerologie an der Humboldt-Universität zu Berlin und leitete als Direktor die Hautklinik der Charité (1950-1962). ${ }^{118}$ Linsers Ziel war eine Reform des SMAD-Befehls Nr. 273.

115 Gertler: Systematische Dermatologie und Grenzgebiete (Anm. 20), S. 1246.

116 Elste: Die SMAD-Befehle 25, 030 und 273 - ihre Bedeutung (Anm. 57), S. 67.

117 Linser K (1948) Vorwort. In: Deutsche Zentralverwaltung für das Gesundheitswesen (Hrsg.) Die gesetzlichen Bestimmungen zur Bekämpfung der Geschlechtskrankheiten in der sowjetischen Besatzungszone Deutschlands. 7-12. AG medizinischer Verlage Berlin.

118 Schneck P (2010) Linser, Karl. In: Wer war wer in der DDR? 5. Ausgabe. Bd 1. Ch. Links Verlag Berlin, S. 622; Böhm B (1992) Leben und Wirken des Arztes Karl Linser (1895 bis 1976), sein Beitrag zur Entwicklung der Dermato-Venerologie und sein Anteil am Wiederaufbau des Gesundheitswesens in der Nachkriegszeit. Diss., Dresden. 
3 Von den geschlossenen Fürsorgeheimen für Geschlechtskranke in der SBZ zu den geschlossenen Venerologischen Stationen in der DDR

Der 1895 in Pforzheim geborene Linser hatte in Karlsruhe das Gymnasium besucht, leistete von 1914 bis 1918 im Ersten Weltkrieg Militärdienst und hatte von 1918 bis 1922 Humanmedizin an den Universitäten Würzburg und Heidelberg studiert. ${ }^{119} 1922$ wurde er mit der Dissertation Ein Beitrag zur Behandlung von Verengungen des Kehlkopfes und der Luftröhre ${ }^{120}$ in Heidelberg zum Doktor der Medizin promoviert. Anschließend war er von 1922 bis 1924 als Assistenzarzt an der Hautklinik der Universität Tübingen, 1924 in Breslau, 1925 an der Hautklinik der Universität Wien und am St.-Louis-Hospital in Paris 1925/1926 tätig. Von 1926 bis 1933 wirkte er als niedergelassener Hautarzt in Dresden. ${ }^{121} \mathrm{Im}$ Jahr 1933 übernahm er die Leitung der Abteilung für Hautkrankheiten am Waldparkkrankenhaus und an der Kinderpoliklinik des Krankenhauses in DresdenJohannstadt. Nach dem Ende des Zweiten Weltkriegs wurde er Chefarzt der Hautklinik des Krankenhauses in Dresden-Friedrichstadt. Im April 1947 erhielt er einen Ruf als Ordinarius für Dermatovenerologie an die Universität Leipzig. Noch im gleichen Jahr folgte die Ernennung zum Präsidenten der Deutschen Zentralverwaltung für das Gesundheitswesen in der SBZ. Anschließend wurde er Leiter der Hauptabteilung Gesund heitswesen im Ministerium für Arbeit und Gesundheitswesen der DDR. Neben seinem Ordinariat an der HumboldtUniversität zu Berlin und seiner Leitung der Hautklinik der Charité war er seit 1951 ärztlicher Direktor des Klinikums Berlin-Buch und Chefarzt der zugehörigen Hautklinik. Karl Linser starb 1976 in Berlin. ${ }^{122}$

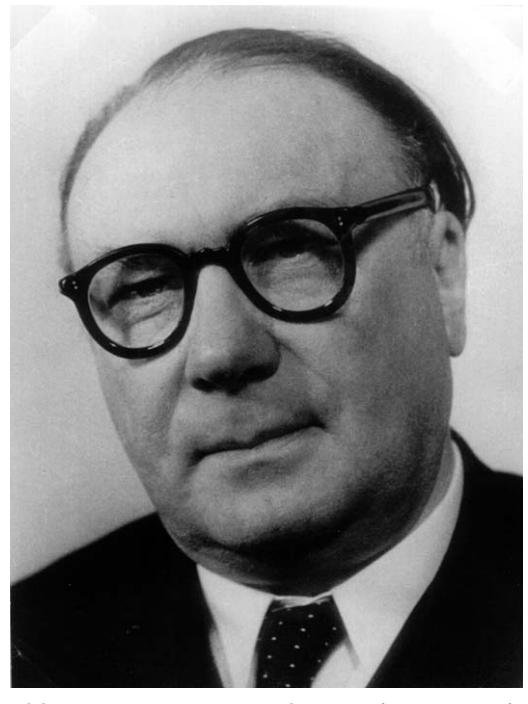

Abb. 5 Porträt von Karl Linser (1895-1979)

119 Schneck: Linser, Karl (Anm. 118).

120 Linser K (1922) Ein Beitrag zur Behandlung von Verengungen des Kehlkopfes und der Luftröhre. Med. Diss., Heidelberg.

121 Schneck: Linser, Karl (Anm. 118).

122 Schneck: Linser, Karl (Anm. 118). 
Linsers Argument für die Reformierung des SMAD-Befehls Nr. 273 lautete, dass sich die Situation auf dem Gebiet der Bekämpfung von Geschlechtskrankheiten seit Ende des Zweiten Weltkriegs geändert hätte und die harte SMAD-Verordnung überflüssig wäre. ${ }^{123}$ In mehreren Briefen wandte sich Linser an den Magistrat von Berlin, das Landesgesundheitsamt und weitere Stellen, um seine Idee voranzubringen. Linsers Reformvorschlag basierte im Wesentlichen auf dem bislang gültigen SMAD-Befehl Nr. 273. Dies geht aus einem Entwurf von Linser für die neue Verordnung hervor. Sein Konzept war eine schreibmaschinengeschriebene Version der bestehenden Verordnung, in der er einzelne Passagen gestrichen bzw. mit Anmerkungen versehen hatte. ${ }^{124}$ Abschaffen wollte Linser unter anderem Teile der $\mathbb{\$} \mathbb{S} 8$ und 9 , in denen es um die Behandlung von Minderjährigen (\$ 8) und die Meldepflicht des Arztes geht (\$ 9). ${ }^{125}$ Mit der Streichung von $\mathbb{9}$ plädierte er gegen die namentliche Meldung von geschlechtskranken Personen, die er wenige Jahre zuvor noch befürwortet hatte. ${ }^{126}$ Weitere Liberalisierungen im Reformvorschlag von Linser sind nicht erkennbar. Erhalten bleiben sollten die Gefängnisstrafen und die Geldstrafen für Ärzte bei Zuwiderhandlung ihrer Pflichten und Bestand hatten nach wie vor die Rechte der Gesundheitsämter. Vor allem aber sollte sich nichts an der zwangsweisen Unterbringung in geschlossenen Venerologischen Stationen ändern. Neben der regen Korrespondenz mit Verwaltungsstellen von Berlin veröffentlichte Linser seine Reformvorschläge auch in dermatologischen Fachzeitschriften. ${ }^{127}$ Trotz des breit angelegten Bemühens, Zustimmung für seine Reformvorschläge zu erhalten, fielen die Reaktionen gemischt aus. Während das Landesgesundheitsamt die Reform begrüßte, signalisierte die Rechtsabteilung des Berliner Magistrats, dass die bisherigen Verordnungen weiterhin Gültigkeit hätten. Zudem hatte das Gesundheitsministerium auf Anfrage seine Zustimmung verweigert. ${ }^{128}$

Erst Anfang der 196oer Jahre wurde der SMAD-Befehl Nr. 273 durch eine Verordnung der DDR-Regierung außer Kraft gesetzt. ${ }^{129}$ Diese „Verordnung zur Verhütung und Bekämpfung von Geschlechtskrankheiten“130 hatte ab 23. Februar

123 LA Berlin: C Rep. 118 - Magistrat von Berlin, Abteilung Gesundheits- und Sozialwesen. Nr. 863: Verordnung zur Bekämpfung der Geschlechtskrankheiten 1955-1956. Schreiben Linsers an den Magistrat von Berlin.

124 LA Berlin: C Rep. 118 - Magistrat von Berlin, Abteilung Gesundheits- und Sozialwesen. Nr. 863: Verordnung zur Bekämpfung der Geschlechtskrankheiten 1955-1956. Verordnung zur Bekämpfung der Geschlechtskrankheiten, Bl. 56-67.

125 LA Berlin: C Rep. 118 - Magistrat von Berlin, Abteilung Gesundheits- und Sozialwesen. Nr. 863: Verordnung (Anm. 123).

126 Vgl. Linser: Vorwort (Anm. 117), S. 10.

127 Linser K (1956) Grundsätzliches über die neue Verordnung zur Bekämpfung der Geschlechtskrankheiten. Das deutsche Gesundheitswesen. Zeitschrift für Medizin XI, 969-977.

128 LA Berlin: C Rep. 118 - Magistrat von Berlin, Abteilung Gesundheits- und Sozialwesen. Nr. 863: Verordnung zur Bekämpfung der Geschlechtskrankheiten 1955-1956. Gutachten der Rechtsabteilung des Berliner Magistrats zur Frage, ob Berlin eine neue Verordnung zur Bekämpfung der Geschlechtskrankheiten benötigt.

129 Willing M (2008) „Sozialistische Wohlfahrt“. Die staatliche Sozialfürsorge in der Sowjetischen Besatzungszone und der DDR (1945-1990). Mohr Siebeck Tübingen, S. 254.

130 Verordnung zur Verhütung und Bekämpfung von Geschlechtskrankheiten (Anm. 23). 
3 Von den geschlossenen Fürsorgeheimen für Geschlechtskranke in der SBZ zu den geschlossenen Venerologischen Stationen in der DDR

1961 Gültigkeit und regelte detailliert alle in der DDR zu ergreifenden Maßnahmen bei Geschlechtskrankheiten. Mit der neuen Verordnung wurden einige der rigiden Bestimmungen des SMAD-Befehls Nr. 273 liberalisiert. ${ }^{131}$ Hintergrund hierfür waren mehrere Entwicklungen: Zum einen war die Anwendung von Penicillin bei der Versorgung Geschlechtskranker seit vielen Jahren eine etablierte Therapie. Seit Mitte der 1940er Jahre konnte beispielsweise die Gonorrhoe durch den Einsatz von Penicillin innerhalb von wenigen Stunden behandelt werden. ${ }^{132}$ So finden sich ab 1947 diverse Schreiben an das Jugend- und Fürsorgeamt Halle (Saale), in denen um die Kostenübernahme für die Behandlung von Geschlechtskranken mit Penicillin gebeten wird. ${ }^{33}$ Diese Schreiben waren meist mit den Namen der Patienten versehen und kamen aus den Einrichtungen, in denen die Geschlechtskranken betreut wurden. Spätestens seit Mitte der 1950er Jahre war das Penicillin auch zur Therapie der Syphilis erprobt und etabliert. ${ }^{134}$ Diese Therapieform führte zu einem Absinken der Infektionsraten, sodass seit Mitte der 195oer Jahre die allgemeine Gefährdung der Bevölkerung nicht mehr als gravierend eingeschätzt wurde. Darüber hinaus zeichnete sich im gleichen Zeitraum mehr und mehr ab, dass Ärzte die Praxis der namentlichen Meldung von Infektionsquellen bei Geschlechtskrankheiten an die Gesundheitsämter infrage stellten. Alternativ diagnostizierten einige Ärzte eine „eitrige Hauterkrankung“, welche nicht den Gesundheitsämtern gemeldet werden musste, aber ebenfalls mit Penicillin behandelt werden konnte. Auf diese Weise konnten sie die namentliche Meldung umgehen. Gründe für dieses Vorgehen waren einerseits ein verbessertes Patient-Arzt-Verhältnis Schutzbedürfnis der Patienten vor beruflicher Benachteiligung und Diffamierung - sowie andererseits die Bestechlichkeit einiger Ärzte. ${ }^{335}$

In der „Verordnung zur Verhütung und Bekämpfung von Geschlechtskrankheiten" waren Stillvorschriften ebenso geregelt wie besondere Untersuchungen bei Schwangeren. In $₫ 2$ wurde festgelegt, wer als geschlechtskrank galt und unter die Bestimmungen der Verordnung fiel: „Geschlechtskrankheiten entsprechend dieser Verordnung sind die übertragbare (infektiöse) Syphilis (Lues venerea), die akute und die chronische Form des Trippers (Gonorrhoe), der weiche Schanker (Ulcus molle) und die Frühform der venerischen Lymphknotenentzündung (Lymphopathia venerea). " ${ }_{136}$ Diese Personen wurden nicht mehr namentlich an die Gesundheitsbehörden gemeldet, wie es der SMADBefehl Nr. 273 vorsah. Vielmehr wurde die namentliche Meldung durch eine chiffrierte Erfassung ersetzt ( $\$ 17)$. ${ }^{137}$ Die Chiffrierung setzte sich aus den Anfangsbuchstaben des Vor- und Zunamens sowie der Angabe des Geschlechts

131 Falck: VEB Bordell. Geschichte der Prostitution (Anm. 71), S. 73.

132 Von Begg-Albensberg: Bericht über den 6. Pflichtkursus (Anm. 94), S. XXIII.

133 StAH: A 2.23 Jugend- und Fürsorgeamt Nr. 106. Bd. 1. Behandlung geschlechtskranker Personen (1928-1950).

134 Falck: VEB Bordell. Geschichte der Prostitution (Anm. 71), S. 74.

135 Falck: VEB Bordell. Geschichte der Prostitution (Anm. 71), S. 74.

136 Verordnung zur Verhütung und Bekämpfung von Geschlechtskrankheiten (Anm. 23), S. 85.

137 Verordnung zur Verhütung und Bekämpfung von Geschlechtskrankheiten (Anm. 23), S. 87. 
3.4 Die „Verordnung zur Verhütung und Bekämpfung von Geschlechtskrankheiten“ vom 23. Februar 1961

und des Geburtsdatums zusammen. Die Daten wurden von den Gesundheitsämtern für statistische Auswertungen verwendet. ${ }^{138}$

Dennoch waren die geschlechtskranken Personen bzw. Krankheitsverdächtigen in $\mathbb{} 10$ verpflichtet, bei der Identifizierung von Infektionsquellen mitzuarbeiten. Sie sollten beim behandelnden Arzt angeben, wer sie angesteckt und wen sie angesteckt haben könnten. Darüber hinaus regelte der $\mathbb{9}$ ein Verbot des Geschlechtsverkehrs: „Der Geschlechtsverkehr und geschlechtsverkehrsähnliche Handlungen sind den Personen gemäß $\mathbb{3}$ verboten. Der Geschlechtsverkehr darf erst nach Erklärung der ärztlichen Unbedenklichkeit wieder ausgeführt werden. Die Kenntnisnahme der Erklärung ist dem Arzt durch Unterschrift zu bestätigen. " ${ }_{139}$ Als Kranke und Krankheitsverdächtige wurden gemäß 3 Personen verstanden, „a) bei denen sich Krankheitserscheinungen finden, die bei Geschlechtskrankheiten vorkommen, b) die nach den Umständen von einem Geschlechtskranken angesteckt sein oder einen anderen mit einer Geschlechtskrankheit angesteckt haben können“. Als dringend krankheitsverdächtig galten Personen, die „a) wiederholt andere mit einer Geschlechtskrankheit angesteckt haben, b) häufig wechselnden Geschlechtsverkehr haben oder häufig wechselnd geschlechtsverkehrsähnliche Handlungen mit anderen Personen vornehmen“. ${ }^{140}$

Eine besondere Einwilligungspflicht zur ärztlichen Behandlung wurde nur bei bestimmten medizinischen Eingriffen gefordert (\$ 8). ${ }^{141}$ Stattdessen kannte die Verordnung ebenso wie der SMAD-Befehl Nr. 273 die zwangsweise Anordnung der Untersuchung und Behandlung bzw. die Einweisung in eine geschlossene Station. Diese Möglichkeiten wurden in $\$ 20$ geregelt, der ein mehrstufiges Verfahren vorsah. Erst am Ende dieses Verfahrens stand bei Missachtung der Anordnungen die Einweisung in eine geschlossene Venerologische Station: „(1) Der Rat des Kreises, Abteilung Gesundheits- und Sozialwesen, kann die Untersuchung oder Behandlung in einer bestimmten Behandlungsstelle oder den Nachweis der Untersuchung oder der Behandlung durch einen vom Patienten zu wählenden berechtigten Arzt befristet verlangen, wenn der Geschlechtskranke oder Krankheitsverdächtige a) eine erforderliche ärztliche Anweisung nicht befolgt, b) sich der ärztlichen Untersuchung, Behandlung oder Nachuntersuchung entzieht, c) entgegen dem Verbot Geschlechtsverkehr oder geschlechtsverkehrsähnliche Handlungen mit anderen Personen ausübt, d) der Überweisung in ein Krankenhaus nicht Folge leistet. (2) Wer sich der angeordneten Untersuchung oder Behandlung entzieht, kann vom Rat des Kreises, Abteilung Gesundheits- und Sozialwesen, zu stationärer Untersuchung oder Behandlung untergebracht werden. (3) Wird dieser Maßnahme nicht nachgekommen, so kann durch den Rat des Kreises, Abteilung Gesund-

138 Falck: VEB Bordell. Geschichte der Prostitution (Anm. 71), S. 74.

139 Verordnung zur Verhütung und Bekämpfung von Geschlechtskrankheiten (Anm. 23), S. 86.

140 Verordnung zur Verhütung und Bekämpfung von Geschlechtskrankheiten (Anm. 23), S. 85.

141 Verordnung zur Verhütung und Bekämpfung von Geschlechtskrankheiten (Anm. 23), S. 86. 
3 Von den geschlossenen Fürsorgeheimen für Geschlechtskranke in der SBZ zu den geschlossenen Venerologischen Stationen in der DDR

heits- und Sozialwesen, die Unterbringung in einer geschlossenen Abteilung für Geschlechtskranke verfügt werden. (4) Der Rat des Kreises, Abteilung Gesundheits- und Sozialwesen, hat die Unterbringung aufzuheben, sobald ihr Zweck erreicht ist. Die Voraussetzungen zur Aufhebung der Unterbringung sind vom Leiter der geschlossenen Abteilung für Geschlechtskranke und vom Rat des Kreises, Abteilung Gesundheits- und Sozialwesen, ständig zu überprüfen." ${ }^{142}$

Darüber hinaus kannte die Verordnung eine Gruppe von Personen, die als „dringend krankheitsverdächtig“ galt. Nach $₫ 3$, Abs. 3 wurden hierunter Personen verstanden, die ,a) wiederholt andere mit einer Geschlechtskrankheit angesteckt haben, b) häufig wechselnden Geschlechtsverkehr haben oder häufig wechselnd geschlechtsverkehrsähnliche Handlungen mit anderen Personen vornehmen". ${ }^{143}$ Für diese Personen sah die Verordnung Sonderregelungen vor. Nach $₫ 18$ mussten alle dringend krankheitsverdächtigen Personen grundsätzlich namentlich gemeldet werden. ${ }^{144}$ Wichtiger war jedoch der $\$ 22$. Dieser war speziell für „dringend Krankheitsverdächtige “vorgesehen und diente im Rahmen der Einweisungspraxis in die geschlossenen Venerologischen Stationen häufig als Begründung für die sofortige Zwangseinweisung. Der $\mathbb{2} 22$ regelte Fragen zur Feststellung der Personalien und der Unterbringung in geschlossenen Venerologischen Stationen: „(1) Der Rat des Kreises, Abteilung Gesundheits- und Sozialwesen, oder dessen Beauftragte können bei den Personen, die als dringend krankheitsverdächtig anzusehen sind ( $\$ 3 \mathrm{Abs} .3$ ), sofort die Personalien feststellen und die Vorlage einer ärztlichen Bescheinigung über den Gesundheitszustand verlangen. (2) Der Rat des Kreises, Abteilung Gesundheits- und Sozialwesen, kann von dringend krankheitsverdächtigen Personen eine ärztliche Untersuchung zur Feststellung von Geschlechtskrankheiten verlangen. Er kann den dringend Krankheitsverdächtigen an eine staatliche Untersuchungs- und Behandlungsstelle verweisen und diese mit der Untersuchung beauftragen. Untersuchungen zur Feststellung des Gesundheitszustandes können wiederholt verlangt werden. (3) Im Krankheitsfalle hat der Rat des Kreises, Abteilung Gesundheits- und Sozialwesen, dringend krankheitsverdächtige Personen in einer staatlichen stationären Behandlungsstelle unterzubringen. Bei Nichtbefolgung dieser Maßnahme oder bei Verdacht, daß dieser nicht Folge geleistet wird, ist die Unterbringung in eine geschlossene Abteilung für Geschlechtskranke zu verfügen. (4) Für die Beendigung der Unterbringung gelten die Bestimmungen des $\mathbb{2 0}$ Abs. 4. “ ${ }^{145}$

Zur Durchsetzung der Verfügungen konnte nach $\mathbb{} 27$ polizeiliche Amtshilfe angefordert werden. So sollte die Polizei etwa bei dringend krankheitsverdächtigen Personen die Personalien feststellen, Kranke oder Krankheitsverdächti-

142 Verordnung zur Verhütung und Bekämpfung von Geschlechtskrankheiten (Anm. 23), S. 87. 143 Verordnung zur Verhütung und Bekämpfung von Geschlechtskrankheiten (Anm. 23), S. 85.

144 Verordnung zur Verhütung und Bekämpfung von Geschlechtskrankheiten (Anm. 23), S. 87.

145 Verordnung zur Verhütung und Bekämpfung von Geschlechtskrankheiten (Anm. 23), S. 88. 
3.4 Die „Verordnung zur Verhütung und Bekämpfung von Geschlechtskrankheiten“ vom 23. Februar 1961

ge der ärztlichen Untersuchung oder Behandlung zuführen oder Personen aufgreifen, die eine ärztliche Untersuchung nicht befolgt hatten. Diese Amtshilfe war an die Voraussetzung gebunden, ,daß die mit der Durchführung der Maßnahmen Beauftragten mit Gewalt bedroht oder tätlich angegriffen werden könnten". ${ }^{146}$

Die Regelungen der „Verordnung zur Verhütung und Bekämpfung von Geschlechtskrankheiten" wiesen eine neue Form des Umgangs mit geschlechtskranken Personen auf. Wer sich zum ersten Mal infizierte, sollte nicht mehr namentlich gemeldet werden. Stattdessen wurde die chiffrierte Weitergabe von Personendaten für die statistische Ausarbeitung in der Verordnung festgeschrieben. Auch die drastischen Strafen, die im SMAD-Befehl Nr. 273 verankert waren, wurden gemildert oder teilweise ganz gestrichen. Gleichzeitig knüpfte die Verordnung an grundsätzliche Normen des vorhergehenden SMADBefehls an. So konnten „dringend Krankheitsverdächtige“, also Personen, die unter dem Verdacht standen, häufig den Geschlechtspartner zu wechseln, nach $\mathbb{2} 22$ sofort in eine geschlossene Einrichtung gebracht werden. Gerade diese Bestimmung bot viel Interpretationsspielraum für die Einweisenden etwa die Organe der Volkspolizei, die nach $\mathbb{2} 7$ bei der Festsetzung „dringend Krankheitsverdächtiger" mitwirken sollten. Auch alle anderen Geschlechtskranken konnten am Ende eines mehrstufigen Verfahrens in eine geschlossene Abteilung zwangseingewiesen werden.

146 Verordnung zur Verhütung und Bekämpfung von Geschlechtskrankheiten (Anm. 23), S. 88. 


\section{Institure-WInenberg \\ Institut füir Strafrecht \\ GES E T Z B L A T T der Deutschen Demokratischen Republik Teil II}

\begin{tabular}{|c|c|c|}
\hline 1961 & Berlin, den 7. März 1961 & Nr. 17 \\
\hline Tag & In halt & Seite \\
\hline 23. 2.61 & Verordnung zur Verhütung und Bekämpfung der Geschlechtskrankheiten.$\ldots \ldots \ldots \ldots$ & 85 \\
\hline 20.2. 61 & $\begin{array}{l}\text { Anordnung über die Berechtigung zum Ausführen von Arbeiten an Energieversor- } \\
\text { gungsanlagen } \ldots \ldots \ldots \ldots \ldots \ldots \ldots \ldots \ldots \ldots \ldots \ldots \ldots \ldots \ldots \ldots \ldots \ldots \ldots \ldots \ldots \ldots \ldots \ldots \ldots \ldots \ldots \ldots \ldots \ldots \ldots \ldots\end{array}$ & 89 \\
\hline
\end{tabular}

\section{Verordnung
zur Verhütung und Bekämpfung \\ der Geschlechtskrankheiten.}

Vom 23. Februar 1961

Verhütung; Untersuchung und Behandlung

$\S 1$

Grundsätze der Verhütung und Bekämpfung der Geschlechtskrankheiten

(1) Der Schutz der Gesundheit der Werktätigen, insbesondere der der Jugend, erfordert die Verhütung und Bekämpfung der Geschlechtskrankheiten auf der und Bekämpfung der Geschlechtskrankheiten auf der Grundlage der neuesten medizinisch-wissenschartlichen Verhütung und Bekämpfung der Geschlechtskrankheiten haben zum Ziel, Neuerkrankungen möglichst zu heiten haben zum Ziel, Neuerkrankungen möglichst zu
verhüten und Frkrankungen frühzeitig zu behandeln verhüten und Frkrankungen frühzeitig zu behandeln sowie Schutzmaßnahmen zu treffen,
tragung der Infektion zu verhindern.

(2) Die Verhütung und Bekämpfung der Geschlechtskrankheiten wird von den Organen des staatlichen krankheiten wird von den Organen des staatlichen Gesundheitswesens geleitet. Der Rat des Bezirkes und der Rat des Kreises hat für eine Koordinierung der Maßnahmen zur Verhütung und Bekämpfung der Geschlechtskrankeiten zu sorgen und insbesondere eng mit der Deutschen Volkspolizei und mit der Staatsund der weitgehenden Verhütung der Ansteckung stützen sie sich besonders auf die Mitwirkung der gesellschaftlichen Organisationen.

(3) Zur fachlichen Unterstützung bei der Durchführung der Maßnahmen des Rates des Bezirkes und de Rates des Kreises, Abteilung Gesundheits- und Sozialwesen, wird ein Facharzt für Haut- und Geschlechtskrankheiten mit der Bezeichnung Bezirks-DermatoVenerologe bzw. Kreis-Dermato-Venerologe entsprechend den Richtlinien eingesetzt.

$$
\$ 2
$$

Geschlechtskrankheiten

Geschlechtskrankheiten entsprechend dieser Verordnung sind die übertragbare (infektiöse) Syphilis (Lue venerea), die akute und die
Trippers (Gonorrhoe), der weiche Schanker (ulcus molle) und die Frühform der venerischen knotenentzündung (Lymphopathia venerea).

$$
\text { s. } 3
$$

Kranke und krankheitsverdächtige Personen

(1) Geschlechtskrank im Sinne der Verordnung sind Personen, die an einer im $\S 2$ bezeichneten Geschlechtskrankheit leiden.

(2) Krankheitsverdächtig sind Personen,

a) bei denen sich Krankheitserscheinungen finden die bei Geschlechtskrankheiten vorkommen,

b) die nach den Umständen von einem Geschlechtskranken angesteckt sein oder einen anderen mit einer Geschlechtskrankheit angesteckt haben können.

(3) Als dringend krankheitsverdächtig gelten Personen, die

a) wiederholt andere mit einer Geschlechtskrankheit angesteckt haben,

b) häufig wechselnden Geschlechtsverkehr haben oder häufig wechselnd geschlechtsverkehrsähnliche Handlungen mit anderen Personen vornehmen.

$$
\S 4
$$

Untersuchungs- und Behandlungspflicht

(1) Wer weiß oder mit der Möglichkeit rechnen muß, daß er geschlechtskrank ist, hat sich unverzüglich ärztlich untersuchen und im Krankheitsfalle ärztlich behandeln $\mathrm{zu}$ lassen sowie sich den notwendigen Nachuntersuchungen oder Nachbehandlungen $\mathrm{zu}$ unterziehen. Die Untersuchung und Behandlung im ansteckenden Stadium gilt als abgeschlossen, wenn hierfür eine schriftliche ärztliche Bescheinigung ausgestellt ist.

(2) Bei Beginn der Untersuchung oder Behandlung erfragt der Arzt, ob bereits zuvor eine ârztliche Untersuchung oder Behandlung stattgefunden und welcher Arzt sie vorgenommen hat. Der Kranke oder Krankheitsverdächtige ist verpflichtet, die erforderlichen heitsverdach tuge ist verptichtet, die erfordertichen Untersuchung oder Behandlung dem bisherigen Arzt inter un uchung oder Behandlung nachzuweisen.

Abb. 6 Gesetzblatt der Deutschen Demokratischen Republik, Teil II, Berlin 1961, „Verordnung zur Verhütung und Bekämpfung von Geschlechtskrankheiten“ 
(3) Ist der Kranke oder Krankheitsverdächtige minderjährig oder fehlen ihm die Voraussetzungen, die Notwendigkeit und Bedeutung der ärztlichen $\mathrm{Ma \beta}$ nahmen und die Ansteckungsgefahr zu erkennen, so ist ein Sorgeberechtigter oder derjenige $\mathrm{zu}$ benachrichtigen, der sonst für sein persönliches Wohl verantwortlich ist.

\section{§ 5}

Berechtigung zur Untersuchung und Behandlung

(1) Die Untersuchung auf Geschlechtskrankheiten und thre Behandlung ist gestattet:

a) Fachärzten für Haut- und Geschlechtskrankheiten,

b) Arzten in einem Fachkrankenhaus für Haut- und Geschlechtskrankheiten oder in einer entsprechenden Fachabteilung mit Beschränkung auf ihre ärztliche Tätigkeit in diesen Behandlungsstellen,

c) anderen Ärzten, die auf Grund ihrer Erfahrungen durch den Rat des Kreises, Abteilung Gesundheits- und Sozialwesen, für Untersuchungen auf Geschlechtskrankheiten und ihre Behandlung zu-

d) ferner, unter Beschränkung auf die Untersuchung und Behandlung bei bestimmten Arten und Erscheinungsformen der Geschlechtskrankheiten, Fachärzten für Kinderkrankheiten für die Behandlung der Syphilis connata der Kinder und Fachärzten für Frauenkrankheiten und Geburtshilfe für die Behandlung der Gonorrhoe bei Frauen.

(2) Årzte, die auf Geschlechtskrankheiten nicht untersuchen und diese nicht behandeln dürfen, Zahnärzte und Heilpraktiker haben bei Verdacht unverzüglich die Uberweisung an einen berechtigten Arzt vorzunehmen.

(3) Nichtärzten ist die Untersuchung und Behandlung der Geschlechtsorgane untersagt.

Empfehlungen für die Untersuchung und Behandlung von Geschlechtskrankheiten

Für die Untersuchung und Behandlung von Geschlechtskrankheiten gibt die Deutsche Akademie der Wissenschaften zu Berlin, Sektion für Dermatologie, wissenschaftliche Empfehlungen heraus. In diesen ist auch das Stadium der Infektiosität im Sinne dieser Verordnung abgegrenzt.

\section{$\S 7$}

Verbot von Maßnahmen der Fernbehandlun

(1) Jede Form der Fernbehandlung von Geschlechts krankheiten und die Erteilung von Ratschlägen für die Selbstbehandlung von Geschlechtskrankheiten ist verboten.

(2) Mittel, Gegenstände oder Verfahren, die Geschlechtskrankheiten heilen oder lindern sollen, dürfen nicht öffentlich oder durch Verbreitung von Schriften, Abbildungen oder Darstellungen angekündigt oder ausgestellt, angeboten, verkauft oder sonst abgegeben werden. Dieses Verbot gilt nicht für Årzte, Apotheker oder für Personen, die mit solchen Mitteln oder Gegenständen erlaubterweise Handel treiben, sowie für Veröffentlichungen in der einschlägigen Fachliteratur.

$$
\S 8
$$

Besondere Einwilligung bei bestimmten ärztlichen$$
\text { Eingriffen }
$$

(1) Die Entnahme von Rückenmarkflüssigkeit, die Cystoskopie, der Ureteren-Katheterismus sowie bestimmte andere vom Ministerium für Gesundheits- wesen festzulegende Eingriffe bedürfen der vorherigen Zustimmung des Patienten. Bei Minderjährigen ist die Einwilligung des Sorgeberechtigten einzuholen.

(2) Lehnen Geschlechtskranke oder Krankheitsverdächtige oder die Sorgeberechtigten Minderjähriger die Vornahme von Eingriffen im Sinne des Abs. 1 ab, so sind sie verpflichtet, dies dem untersuchenden oder behandelnden Arzt schriftlich zu bestätigen.

Verbot des Geschlechtsverkehrs

Der Geschlechtsverkehr und geschlechtsverkehrsähnliche Handlungen sind den Personen gemäß $\$ 3$ verboten. Der Geschlechtsverkehr darf erst nach Erklärung der ärztlichen Unbedenklichkeit wieder ausgeführt der ärztlichen Unbedenklichkeit wieder ausgeführt
werden. Die Kenntnisnahme der Erklärung ist dem werden. Die Kenntnisnahme der Erk
Arzt durch Unterschrift zu bestätigen.

\section{Arztliche Feststellungen $\$ 10$}

Petstellungen der Ansteckungsmöglichkeiten (1) Der behandelnde Arzt hat den Kranken oder Krankheitsverdächtigen eingehend $\mathrm{zu}$ befragen, wer ihn angesteckt haben und wer von ihm angesteckt sein kann.

(2) Der Kranke oder Krankheitsverdächtige ist verpflichtet, die erforderlichen Auskünfte nach bestem Wissen zu geben und bei der Feststellung jeder Person, die ihn angesteckt haben oder die von ihm angesteckt sein kann, zumutbare Hilfe zu leisten.

$$
\S 11
$$

\section{Besondere Untersuchung bei Schwangeren}

Eine Schwangere, die an Syphilis erkrankt war, hat sich, sobald sie von der Schwangerschaft weiß, vorbeugend durch einen dazu berechtigten Arzt untersuchen und, wenn nötig, behandeln zu lassen.

\section{Besondere Untersuchung und Behandlung von Neugeborenen}

Ein Neugeborenes, dessen Mutter an Syphilis erkrankt war oder ist und deren Behandlung ungenügend war, ist in den ersten 6 Lebensmonaten vorbeugend zu behandeln.

$$
\S 13
$$

\section{Stillvorschriften}

(1) Eine Frau, die ein fremdes Kind stillt oder ihre Milch zur Ernährung anderer Kinder spendet, muß im Besitz eines Zeugnisses eines berechtigten Arztes sein, daß keine Anhaltspunkte für das Vorliegen einer Geschlechtskrankheit bestehen. Das Zeugnis muß kurz vor dem ersten Stillen oder vor der ersten Milchspende ausgestellt sein und darf in keinem Falle älter als 1 Monat sein. Ein Auftrag zum Stillen bzw. die Abnahme der Milchspende darf nur bei Vorlage dieses Zeugnisses erfolgen.

(2) Eine Frau, die ein fremdes Kind stillt oder ihre Milch zur Ernährung anderer Kinder spendet, darf nicht an einer Syphilis erkrankt gewesen sein.

(3) Der Sorgeberechtigte darf eine Frau mit dem Stillen seines Kindes erst dann beauftragen, wenn er im Besitz eines ärztlichen Zeugnisses ist, daß der Frau aus dem Stillen des Kindes keine Gesundheitsgefahr erwächst.

(4) Ein an Syphilis erkranktes Kind darf nur von (4) Ein an Syphilis erkrank
seiner Mutter gestillt werden.

Fortsetzung von Abb. 6 Gesetzblatt der Deutschen Demokratischen Republik, Teil II, Berlin 1961, „Verordnung zur Verhütung und Bekämpfung von Geschlechtskrankheiten" 


\section{ufklärungspflicht $\$ 14$ \\ Ubergabe eines Kindes} in Pflege

Wer ein geschlechtskrankes Kind in Pflege gibt, hat zuvor den Pflegeeltern von dessen Geschlechtskrankheit oder von einem ärztlich festgestellten Verdach aue eine solche Krankheit Kenntnis zu geben. auf eine gilt auch für ein Kind, das an einer Syphilis erkrankt war oder warde.

\section{\$ 15}

Wer an einer Geschlechtskrankheit leidet oder an Syphilis gelitten hat, darf kein Blut spenden.

$$
\text { \$ } 16
$$

Pflicht zur Belehrung

Der berechtigte Arzt hat den Patienten bei Krankheitsverdacht, bei Aufnahme der Behandlung und nach Beseitigung der Infektiosität über sein Verhalten $\mathrm{zu}$ belehren und ein Merkblatt auszuhändigen. Die Belehrung und Aushändigung des Merkblattes sind vom Patienten schriftlich zu bestätigen.

\section{\$ 17
Meldepplich}

(1) Xrzte, Zahnärzte und Hebammen haben innerhalb (1) Arze, Zahnaraten sundheits- und Soziaiwesen,

a) Geschlechtskranke oder krankheitsverdächtige Personen,

b) Personen, die Geschlechtskranke oder Krankheitsverdächtige angesteckt haben oder die von Geschlechtskranken oder Krankheitsverdächtigen angesteckt sein könrien,

c) Schwangere, die an Syphilis erkrankt sind oder waren,

d) neugeborene Kinder einer an Syphilis erkrankten oder erkrankt gewesenen Frau

unter Deckbezeichnung ohne Namensnennung zu melden.

(2) Die Frist gemäß Abs. 1 beginnt im Falle der Buchstaben a bis c mit der Feststellung einer Gechlechtskrankheit oder eines Krankheitsverdachtes, im Falle des Buchst. d mit der Geburt.

$$
\$ 18
$$

\section{Namentliche Meldung}

(1) Namentlich zu melden ist ein Geschlechtskranker oder Krankheitsverdächtiger, der

a) sich trotz der Verpflichtung ärztlich nicht untersuchen oder behandeln läßt oder sich nicht der Untersuchung bzw. Behandlung bis zum Abschluß unterzieht,

b) bei Beginn der Untersuchung oder Behandlung nicht angibt, von welchem Arzt er zuvor untersucht oder behandelt worden ist,

c) den ärztlichen Anordnungen hinsichtlich der Untersuchung und Behandlung, der Überweisung durch eine zur Untersuchung oder Behandlung durch eine zur Untersuchung oder Behandlung Arzt oder der ärztlichen Uberweisung zur statioArzt oder der ärztlichen Uberweisung

d) sich entgegen dem Verbot des Geschlechtsverkehrs oder geschlechtsverkehrsähnlichen Handlungen nicht enthält, e) erforderliche Auskünfte über die Ansteckungs möglichkeiten dem berechtigten Arzt nicht gib oder vom Arzt zur Untersuchung richt aufgefordert werden kann,

f) entgegen dem Verbot Blut spendet,

g) durch die berufliche Tätigkeit eine erhöhte Ansteckungsgefahr bietet,

h) als dringend krankheitsverdächtig gilt.

(2) Wenn mehrfache Meldungen auf einen dringend Krankheitsverdächtigen schließen lassen, so kann der Rat des Kreises, Abteilung Gesundheits- und Sozialwesen, eine namentliche Meldung verlangen.

(3) Der eine Schwangerschaft feststellende Arzt hat eine Schwangere, wenn sie sich nicht gemäß § 11 vorbeugend untersuchen und behandeln läßt, namentlich zu melden. Desgleichen hat der Geburtshilfe leistende Arzt oder die Hebamme ein Neugeborenes namentlich zu melden, wenn der Sorgeberechtigte die vorbeugende ärztliche Behandlung eines Neugeborenen gemäß § 12 nicht vornehmen läßt.

\section{Oberweisung zur stationären Behandlun}

(1) Ein Kranker oder Krankheitsverdächtiger ist vom Arzt zur stationären Untersuchung oder Behandlung in ein Krankenhaus zu überweisen, wenn dies zur Feststellung oder Behandlung einer Geschlechtskrankheit oder wegen der Gefahr ihrer Weiterverbreitung erforderlich ist.

(2) Bei folgenden Krankheitserscheinungen durch die Erreger der Syphilis bzw. des Trippers

a) Säuglingssyphilis,

b) Vulvovaginitis gonorrhoica infantum,

c) Ophthalmo-Blenorrhoe

ist der Arzt verpflichtet, die Uberweisung zur statio nären Behandlung vorzunehmen. Der Rat des Kreises, Abteilung Gesindheits- und Sozialwesen, kann au ärztlichen Antrag hiervon Befreiung bewilligen.

\section{$\S 20$}

\section{Anordnung der Untersuchung und Behandlung}

(1) Der Rat des Kreises, Abteilung. Gesundheits- und Sozialwesen, kann die Untersuchung oder Behandlung in einer bestimmten Behandlungsstelle oder den Nachweis der Untersuchung oder der Behandlung durch einen vom Patienten zu wählenden berechtigten Arzt befristet verlangen, wenn der Geschlechtskranke oder Krankheitsverdächtige

a) eine erforderliche ärztliche Anweisung nicht befolgt,

b) sich der ärztlichen Untersuchung, Behandlung ader Nachuntersuchung entzieht

c) entgegen dem Verbot Geschlechtsverkehr oder geschlechtsverkehrsähnliche Handlungen mit anderen Personen ausübt,

d) der Uberweisung in ein Krankenhaus nicht Folge leistet.

(2) Wer sich der angeordneten Untersuchung oder Behandlung entzieht, kann vom Rat des Kreises, $\mathrm{Ab}$ teilung Gesundheits- und Sozialwesen, zu stationäre Untersuchung oder Behandlung untergebracht werden.

(3) Wird dieser Maßnahme nicht nachgekommen, so kann durch den Rat des Kreises, Abteilung Gesundheits- und Sozialwesen, die Unterbringung in einer geschlossenen Abteilung für Geschlechtskranke verfügt werden.

Fortsetzung von Abb. 6 Gesetzblatt der Deutschen Demokratischen Republik, Teil II, Berlin 1961, „Verordnung zur Verhütung und Bekämpfung von Geschlechtskrankheiten“ 
(4) Der Rat des Kreises, Abteilung Gesundheits- und Sozialwesen, hat die Unterbringung aufzuheben, sobald ihr Zweck erreicht ist. Die Voraussetzungen zur Aufhebung der Unterbringung sind vom Leiter der geschlossenen Abteilung für Geschlechtskranke und vom Rat des Kreises, Abteilung Gesundheits- und Sozialwesen, ständig zu überprüfen.

\section{$\S 21$}

\section{Aufzeichnungspflicht}

Uber die Untersuchungen und Behandlungen von Geschlechtskrankheiten sowie über spezielle Fürsorge bei Geschlechtskranken oder Krankheitsverdächtigen sind Aufzeichnungen in der vom Ministerium für Gesundheitswesen vorgeschriebenen Form anzufertigen und aufzubewahren.

\section{$\S 22$}

\section{Besondere Maßnahmen bei dringend}

\section{Krankheitsverdächtigen}

Krankheitsverdãchtigen
(1) Der Rat des Kreises, Abteilung Gesundheits- und Sozialwesen, oder dessen Beauftragte können bei den Personen, die als dringend krankheitsverdäcintig anzusehen sind ( $\$ 3$ Abs. 3), sofart die Personalien feststellen und die Vorlage einer ärztlichen Bescheinigung uber den Gesundheitszustand verlangen.

(2) Der Rat des Kreises, Abteilung Gesundheits- und Sozialwesen, kann von dringend krankheitsverdächSozialwesen, kann von dringend krankheitsverdächtigen Personen eine arztliche Untersuchung zur Feststellung von Geschlechtskrankheiten verlangen. E kann den dringend Krankheitsverdächtigen an ein staatliche Untersuchungs- und Behandlungsstelle verweisen und diese mit der Untersuchung beauftragen. Untersuchungen zur Feststellung des Gesundheitszustandes können wiederholt verlangt werden.

(3) Im Krankheitsfalle hat der Rat des Kreises, Abteilung Gesundheits- und Sozialwesen, dringend krankheitsverdächtige Personen in einer staatlichen stationaren Behandlungsstelle unterzubringen. Be Nichtbefolgung dieser Maßnahme oder bei Verdacht, daß dieser nicht Folge geleistet wird, ist die Unter-
bringung in eine geschlossene Abteilung für Gebringung in eine geschloss
schlechtskranke zu verfügen.

(4) Für die Beendigung der Unterbringung gelten die Bestimmungen des $\$ 20$ Abs. 4

$$
\text { \& } 23
$$

Maßnahmen bei anderen Persone

Bei Personen, welche an Orten, die für den Aufenthalt dringend Krankheitsverdächtiger bekannt sind, angetroffen werden und die den Verdacht des Umgangs angetroffen werden und die den Verdacht des Umgangs
mit solchen Personen erregen, können der Rat des Kreises, Abteilung Gesundheits- und Sozialwesen, oder dessen Beauftragte

a) die Personalien sofort feststellen,

b) die unverzügliche Vorlage einer ärztlichen $\mathrm{Be}-$ scheinigung über den Gesundheitszustand und bei Nichtbefolgung die Untersuchung in einer staatlichen Behandlungsstelle verlangen.

\section{$\stackrel{824}{\text { Sozialheime }}$}

Die geeignete Betreuung dringend Krankheitsverdächtiger über $18 \mathrm{Jahre}$ in Sozialheimen kann durch die Räte der Kreise, Abteilung Gesundheits- und Sozialwesen, mit Zustimmung der aufzunehmenden Person erfolgen. Die Unterbringung hat zum Ziel, durch erzieherische Arbeit und geregelte Lebensweise eine Besserung der Untergebrachten zu erreichen und die Rückführung in die Gesellschaft zu fördern.

\section{$\S 25$}

\section{Berufsbeschränkungen}

Der Rat des Kreises, Abteilung Gesundheits- und Sozialwesen, kann zur Verhütung der Ansteckung Berufsbeschränkungen befristet oder unbefristet aussprechen.

\section{$\S 26$}

Verfügungen und Beschwerden

(1) Verfügungen des Rates des Kreises, Abtellung (1) Verfügungen des Rates des Kreises, Abtellung Gesundheits- und Sozialwesen, sind schriftlich zu er-
lassen, $\mathrm{zu}$ begründen, mit Rechtsmittelbelehrung zu làssen, $\mathrm{zu}$ begründen, mit Rechtsmittelbeleht
versehen und dem Betroffenen zuzustellen.

(2) Gegen eine Verfügung des Rates des Kreises, Abteilung Gesundheits- und Sozialwesen, gemä3 den Bestimmungen des $\S 20$ Absätze 1 bis 3 , § 22 Abs. 3, $\$ 25$ oder gegen die Zurücknahme einer Zulassung ge$\$ 25$ oder gegen die Zurücknahme einer Zulassung geder Beschwerde an den Rat des Kreises, Abteilung Geder Beschwerde an den Rat des Kreises, Abteilung Gesundheits- und Sozialwesen, schriftlich einzulegen oder mündlich zu Protokoll zu erklären und gleichzeitig zu
begründen.

(3) Wird die Beschwerde für begründet gehalten, so ist dieser binnen 1 Woche nach Eingang der Beschwerde abzuhelfen, anderenfalls ist sie an den Rat des Bezirkes, Abteilung Gesundheits- und Sozialwesen, zirkes, Abteilung Gesundheits- und Sozialwesen,
weiterzuleiten. Uber die Beschwerde ist binnen 2 Woweiterzuleiten. Uber die Beschwerde ist binnen 2 Wo-
chen nach Eingang endgültig zu entscheiden. Diese chen nach Eingang endgültig zu entscheiden. Diese
Entscheidungen sind schriftlich zu erteilen und zu beEntscheich
gründen.

(4) Die Beschwerde hat keine aufschiebende Wirkung. Das für die Beschwerdeentscheidung zuständige Organ kann jedoch die Durchführung der angeordneten Masnahmen aussetzen.

\section{$\S 27$
Durchsetzung von verfügungen und polizeiliche Amtshilfe}

(1) Werden Maßnahmen zur ordnungsmäßigen Durchführung getroffener Verfügungen

a) gegen einen Kranken oder Krankheitsverdächgegen einen Kranken oder Krankheitsverdächlung gemäß $\$ 20$ Absätzen 1 bis 3

b) gegen eine dringend krankheitsverdächtige Person zur Feststellung der Personalien oder gegen eine andere Person zur ärztlichen Untersuchung oder Behandlung gemäß \& 22 Absätzen 1 bis 3 ,

c) gegen andere Personen zur Feststellung der Personalien oder zur ärztlichen Untersuchung

nicht befolgt, können diese entsprechend durchgesetzt werden.

(2) Die Organe der Deutschen Volkspolizei leisten bei der Durchführung dieser Maßnahmen Amtshilfe, wenn den Umständen nach zu erkennen ist, daß die mit der Durchführung der Maßnahmen Beauftragten mit Gewait bedroht oder tätlich angegriffen werden könnten.

Straf- und Ordnungsstrafbestimmungen

$$
\S 28
$$

Ordnungsstrafen

(1) Mit einer Ordnungsstrafe bis zu $500 \mathrm{DM}$ kann bestraft werden, wer

a) als Kranker oder Krankheitsverdächtiger seine Pflichten gemäß \$ 4 Abs. 1 oder 2, § 10 Abs. 2 oder \& 20 Abs. 1 oder 2 verletzt,

Fortsetzung von Abb. 6 Gesetzblatt der Deutschen Demokratischen Republik, Teil II, Berlin 1961, „Verordnung zur Verhütung und Bekämpfung von Geschlechtskrankheiten" 


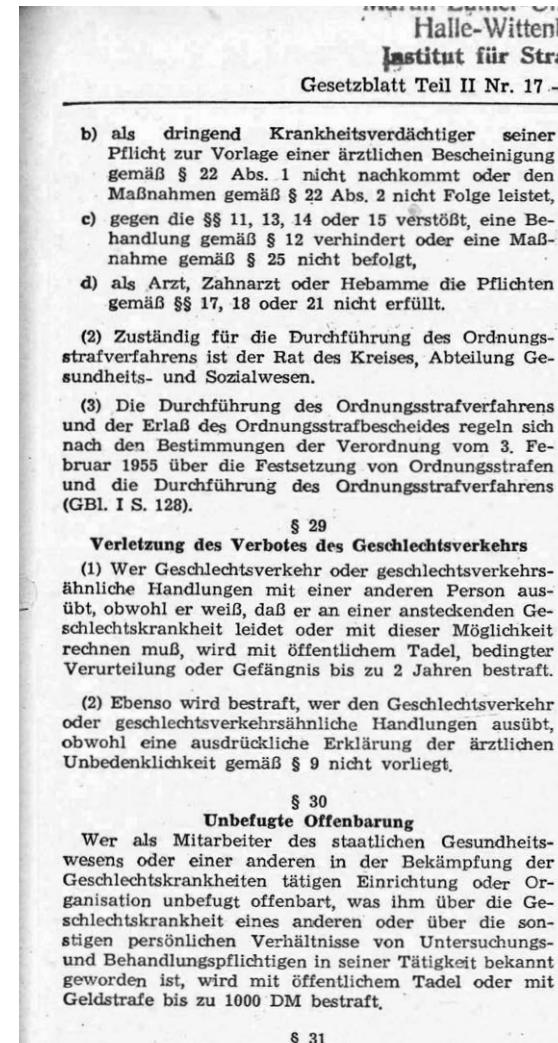
Unberechtigte Untersuchung und Behandlung sowie Fernbehandlung

(1) Wer, ohne Arzt zu sein, die Geschlechtsorgane eines Menschen untersucht oder behandelt, wird mit öffentlichem Tadel, mit Geldstrafe bis zu $1000 \mathrm{DM}$, mit bedingter Verurteilung oder mit Gefängnis bis zu bedingter Verurte

(2) Ebenso wird bestraft, wer eine Fernbehandlung von Geschlechtskrankheiten durchführt oder wer öffentlich oder durch Verbreitung von Schriften, Abbildungen oder Darstellungen Ratschläge für die Selbstbehandlung von Geschlechtskrankheiten erteilt.

$$
\text { III. }
$$

Schlußbestimmungen

$$
\text { \$ } 32
$$

Durchfïhrungsbestimmungen

Durchíührungsbestimmungen erläßt der Minister für Gesundheitswesen.

$$
\begin{gathered}
833 \\
\text { Inkraftreten }
\end{gathered}
$$

(1) Diese Verordnung tritt am 10. März 1961 in Kraft.
(2) Gleichzeitig treten

a) die Verordnung vom 11. Dezember 1947 zur Be kämpfung der Geschlechtskrankheiten unter der deutschen Bevölkerung in der sowjetischen $\mathrm{B}$ satzungszone Deutschlands (ZVOBI. 1948 S. 44),

b) die Erste Verordnung vom 30. Juli 1948 zur Durchführung und Ergänzung der Verordnung zur Bekämpfung der Geschlechtskrankheiten unter der deutschen Bevölkerung in der sowjetischen Besatzungszone Deutschlands (ZVOBl. S. 526)

außer Kraft.

Berlin, den 23. Februar 1961

Der Ministerrat

der Deutschen Demokratischen Republik

Der Minister für Gesundheitswesen

$\mathbf{R}$ a u

Stellvertreter S ef $\mathbf{r i n}$

Stellvertreter
des Vorsitzenden
des Vorsitzenderter

$\begin{array}{ll}\text { des Vorsitzenden } & \text { des Vorsitzenden } \\ \text { des Ministerrates } & \text { des Ministerrates }\end{array}$

Anordnung
über die Berechtigung zum Ausführen von Arbeiten an Energieversorgungsanlagen.

Vom 20. Februar 1961

Zur Gewährleistung der sach- und fachgerechten Ausführung von Arbeiten an Energieversorgungsarlagen wird auf Grund des $\$ 9$ der Verordnung vom 17. März 1960 über die Planung und Leitung. der Energiewirtschaft (GBI I S. 211) im Einvernehmen mit den den Leitern der zuständigen zentralen staatlichen
Organe folgendes angeordnet:

$$
\S 1
$$

\section{Begriffsbestimmungen}

(1) Energieversorgungsanlagen im Sinne dieser Anordnung sind

a) elektrische Starkstromanlagen,

b) Gasanlagen,

c) Fernwärmeanlagen,

die der Verteilung und Abnahme von Elektroenergie, Gas oder Wärme dienen.

(2) Das Ausführen von Arbeiten an Energieversorgungsanlagen umfaßt die Errichtung von Energieversorgungsanlagen und die Vornahme von Arbeiten an diesen Anlagen.

(3) Berechtigte Hersteller sind Bürger und juristische Personen, die nach den Bestiminungen dieser Anordnung zur Ausführung von Arbeiten an Energieversorgungsanlagen berechtigt sind.

\section{$\S 2$}

Erteilung der Berechtigun

(1) Bürger und juristische Personen (z. B. Betriebe und Genossenschaften), deren wirtschaftlicher Z und Given tie Ausführung von ausschlieblich oder tellweise auf die Aung von Arbeiten an Energieversorgungsanlagen gerichtet ist, sind verpflichtet, bei dem örtlich für sie zuständige VEB Energieversorgung (EVB) unter Nachweis der in dieser Anordnung festgelegten persönlichen und technischen Voraussetzungen die Berechtigung zum Ausführen der genannten Arbeiten zu beantragen.

(2) Der EVB erteilt bei Vorliegen der geforderten Voraussetzungen durch Ausstellung eines Ausweises die Berechtigung zum Ausführen der Arbeiten.

Fortsetzung von Abb. 6 Gesetzblatt der Deutschen Demokratischen Republik, Teil II, Berlin 1961, „Verordnung zur Verhütung und Bekämpfung von Geschlechtskrankheiten“" 